\title{
Revision of the African shieldbug genus Afrius Stål, 1870 (Hemiptera: Heteroptera: Pentatomidae: Asopinae)
}

\author{
Talita ROELL ${ }^{1, *}$, Valérie A. LEMAÎTRE ${ }^{2}$ \& Michael D. WEBB ${ }^{3}$ \\ ${ }^{1}$ Universidade Federal do Rio Grande do Sul, Instituto de Biociências, Departamento de Zoologia, \\ Av. Bento Gonçalves 9500, Porto Alegre, 91501-970, RS, Brazil. \\ ${ }^{2,3}$ Natural History Museum, London, Department of Life Sciences, \\ Cromwell Road, London SW7 5BD, UK. \\ *Corresponding author: tali.roell@gmail.com \\ ${ }^{2}$ Email: v.lemaitre@nhm.ac.uk \\ ${ }_{3}^{3}$ Email: m.webb@nhm.ac.uk

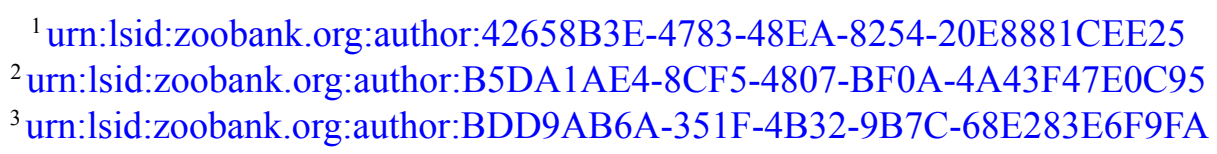

\begin{abstract}
The African shieldbug genus Afrius Stål, 1870 is revised. Cantheconidea migratoria Distant, 1913 and A. williamsi Miller, 1952 are proposed as junior synonyms of A. (Subafrius) flavirostrum (Signoret, 1861) whereas Canthecona marmorata Dallas, 1851, Canthecona annulipes Dallas, 1851 and A. rubromarginatus Bergroth, 1903 are proposed as junior synonyms of $A$. (Afrius) purpureus (Westwood, 1837) based on the general morphology and genitalia of the species. The three valid species, viz. A. (Subafrius) flavirostrum, A. (Afrius) kolleri Schouteden, 1911 and A. (Afrius) purpureus, are redescribed with details of male and female genitalia morphology, and a lectotype is designated for A. (Afrius) kolleri. A key to identify the species as well as an update of the geographical distribution for each species are provided, including new records for A. (Afrius) purpureus.
\end{abstract}

Keywords. Stink bugs, predation, taxonomy, polymorphism, Ethiopia.

Roell T., Lemaître V.A. \& Webb M.D. 2019. Revision of the African shieldbug genus Afrius Stål, 1870 (Hemiptera: Heteroptera: Pentatomidae: Asopinae). European Journal of Taxonomy 520: 1-44.

https://doi.org/10.5852/ejt.2019.520

\section{Introduction}

The subfamily Asopinae is the only predatory subfamily of Pentatomidae. It is an important economic group, containing many species used as biological control agents for pest management (Grazia et al. 2015). The asopines have a worldwide distribution and are recognized mainly by the robust labium and, in the male genitalia, by the presence of genital plates and a thecal shield (Thomas 1992, 1994). The asopine genus Afrius Stål, 1870 is distributed throughout Africa and its species have been considered potential biological control agents for insects injurious to plantations in different regions of Africa (e.g., Miller 1952; Sileshi et al. 2004). Afrius was created as a subgenus of Cimex Linnaeus, 1758 
by Stål (1870) with three species, Cimex (Afrius) figuratus (Germar, 1838), C. (Afrius) purpureus (Westwood, 1837) and C. (Afrius) flavirostris (Signoret, 1861), characterized within the inclusive genus by abdominal lateral margins convex and anterior femora armed with spines. In his synopsis of the Old World asopine genera, Thomas (1994) presented a diagnosis of the genus and mentioned the lack of a species identification key and the necessity of revisionary studies on the genus. After examining type and other material of all species, we here provide a revision of the genus, with habitus and genitalia figures and descriptions of each species, new synonymies and new records.

\section{Material and methods}

Type and other material were examined and photographed at The Natural History Museum, London, UK (NHMUK), the Musée royal de l'Afrique centrale, Belgium (RMCA), the Museum für Naturkunde Berlin, Germany (MFNB), the Muséum national d'Histoire naturelle, Paris, France (MNHN), the University Museum of Natural History in Oxford, UK (OUMNH), and the Federal University of Rio Grande do Sul, Porto Alegre, Brazil (UFRG). Extra material was received from the American Museum of Natural History, USA (AMNH), David A. Rider Collection, USA (DARC), the Entomologisches Museum Insekten Dauerausstellung, Geyer, Germany, and the National Museum of Prague, Czech Republic (NMPC). Photographs were received from the Naturhistorisches Museum Wien, Austria (NHMW), and the Naturhistoriska Riksmuseet, Stockholm, Sweden (NHRS). The examined material is listed in Table 1, and the geographic coordinates in decimal degrees were taken from the Google Earth program (ver. 7: https://www.google.com/earth/) and from the 'GeoNames' website (http://www.geonames.org) when

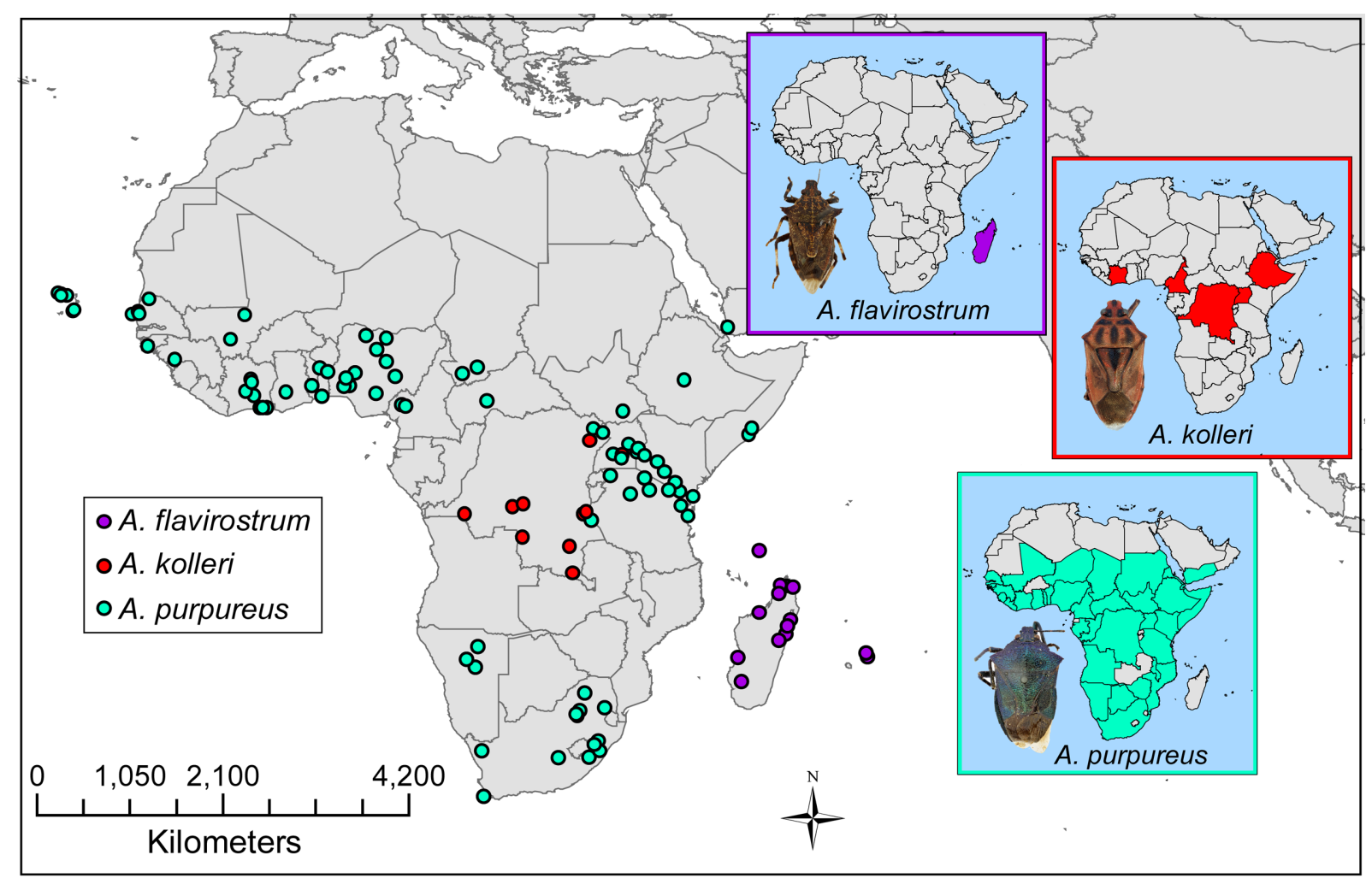

Fig. 1. Geographic distribution of the genus Afrius Stål, 1870 throughout Africa. The species points of occurrence and their dispersion in different countries are represented with colours: purple $=A$. (Subafrius) flavirostrum (Signoret, 1861); red = A. (Afrius) kolleri Schouteden, 1911; green = A. $($ Afrius) purpureus (Westwood, 1837). The countries highlighted for each species on the right part of the map are based on data from literature. The points marked for each species are from literature and labels information. 


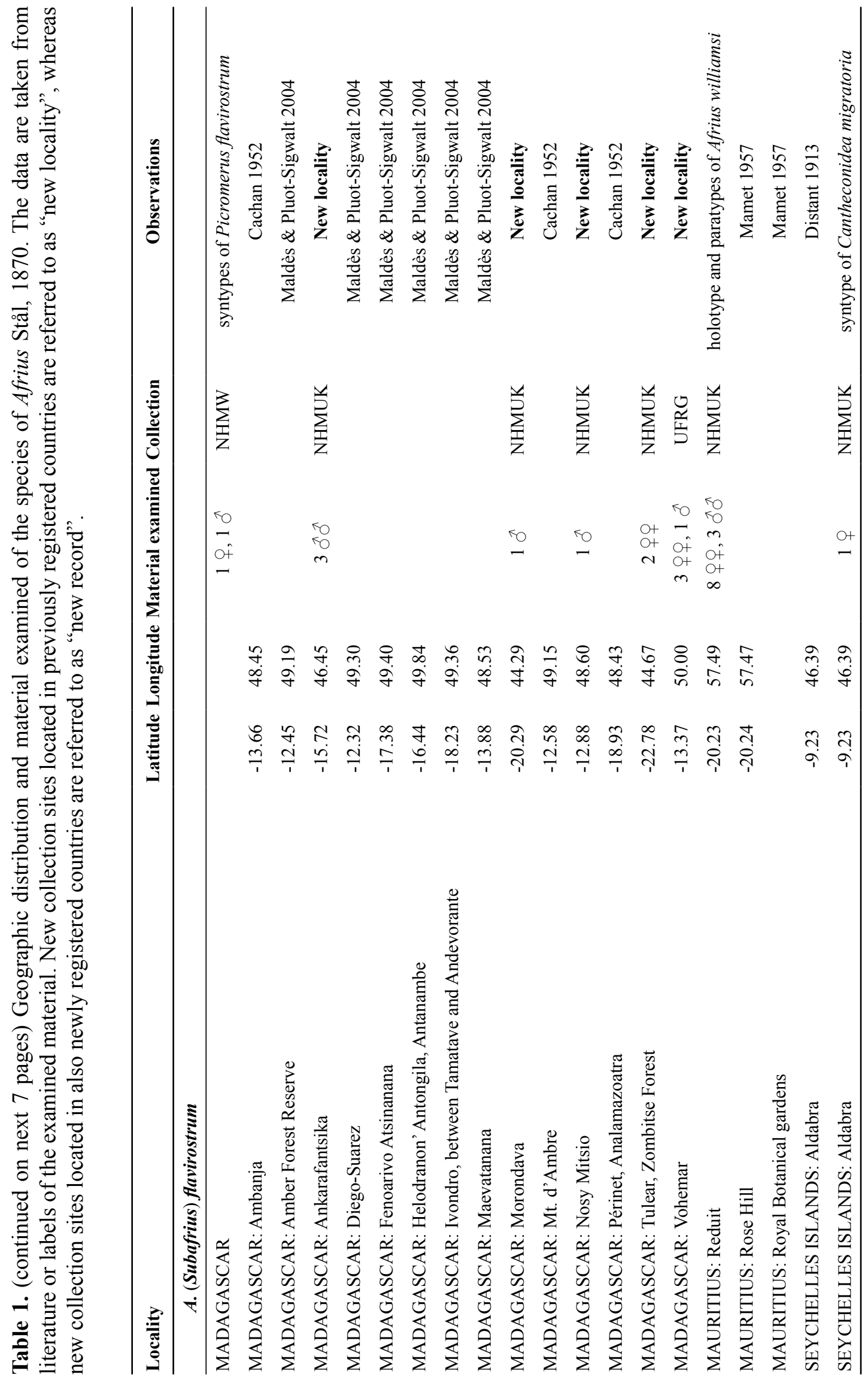




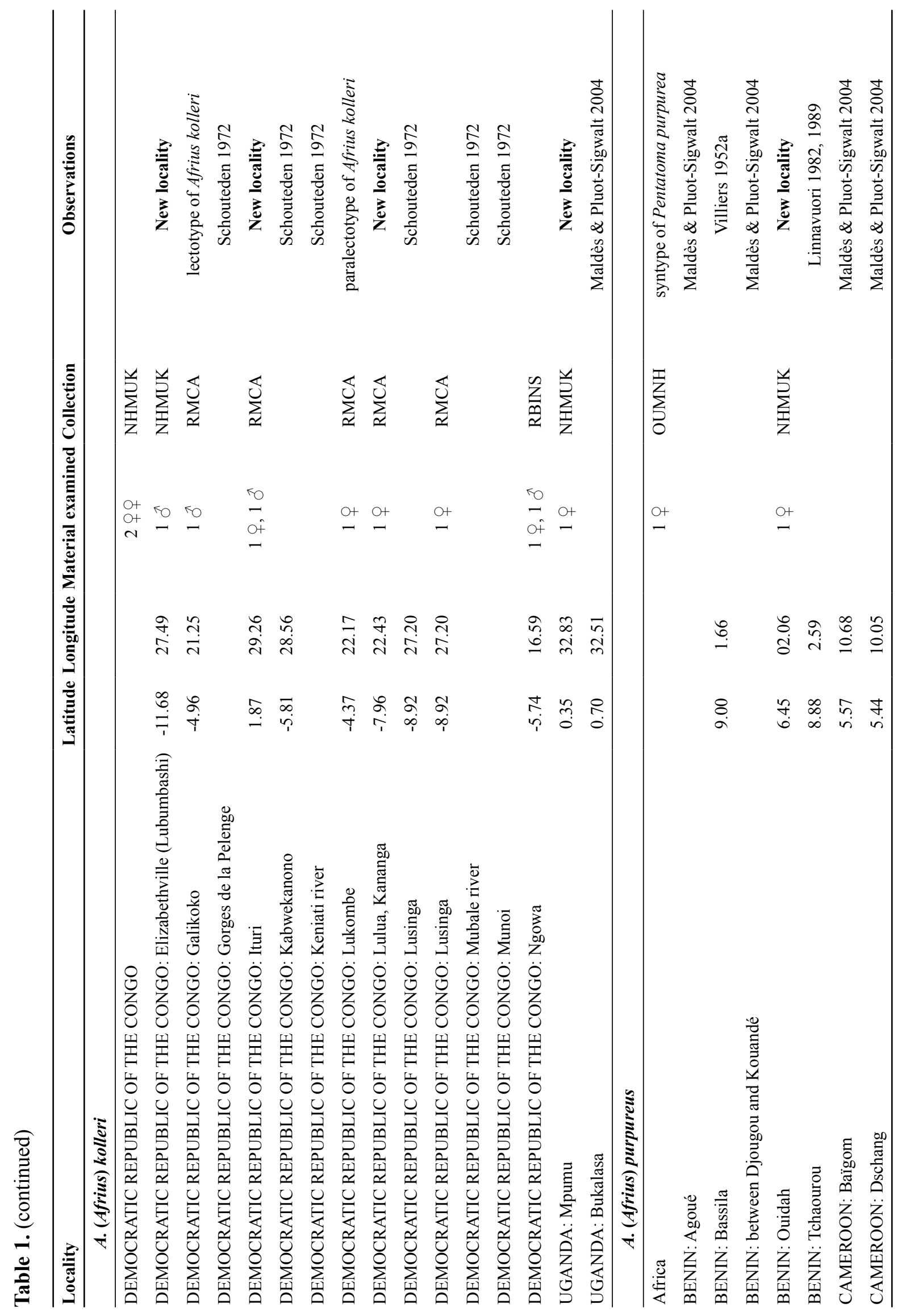




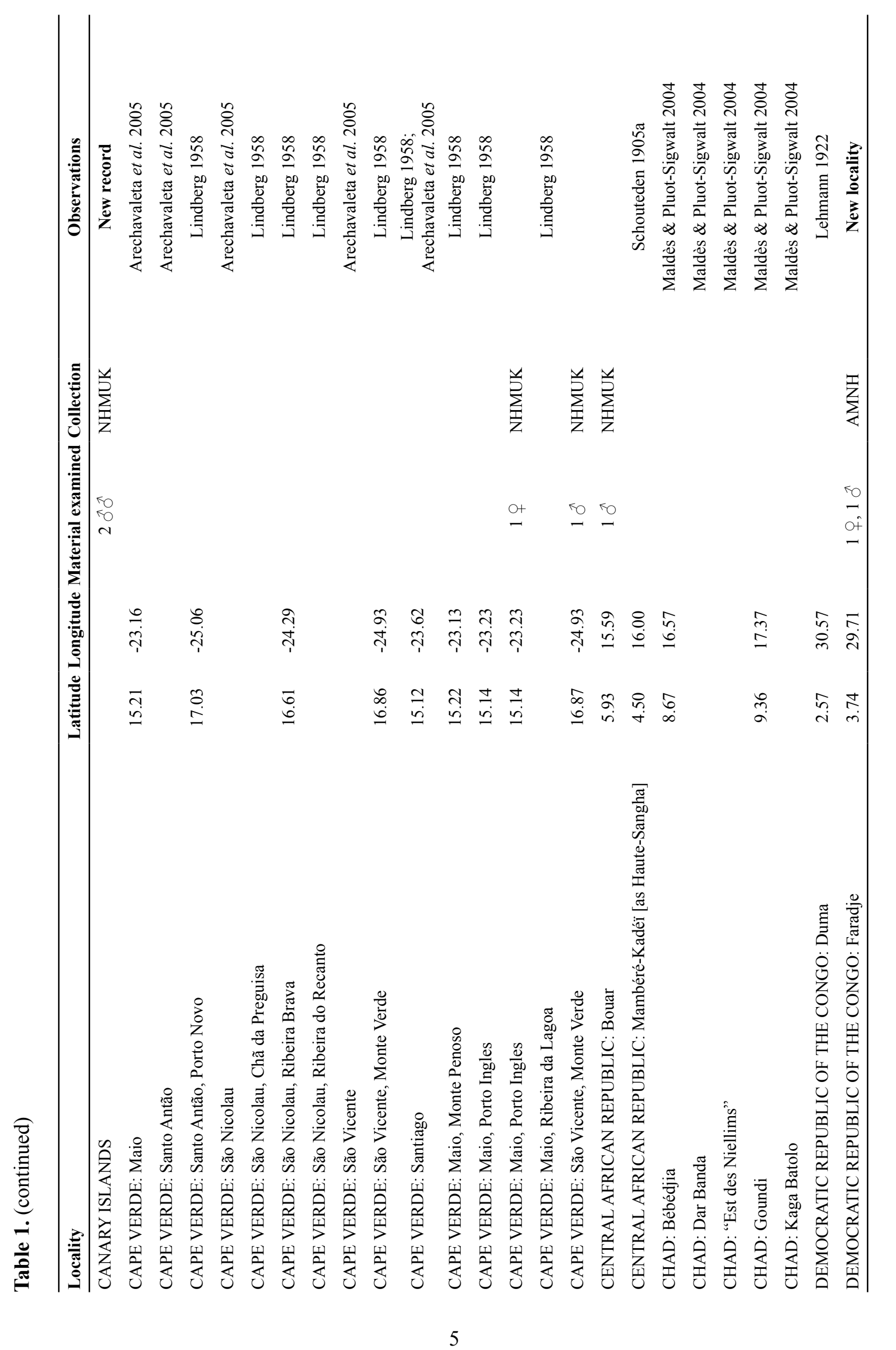




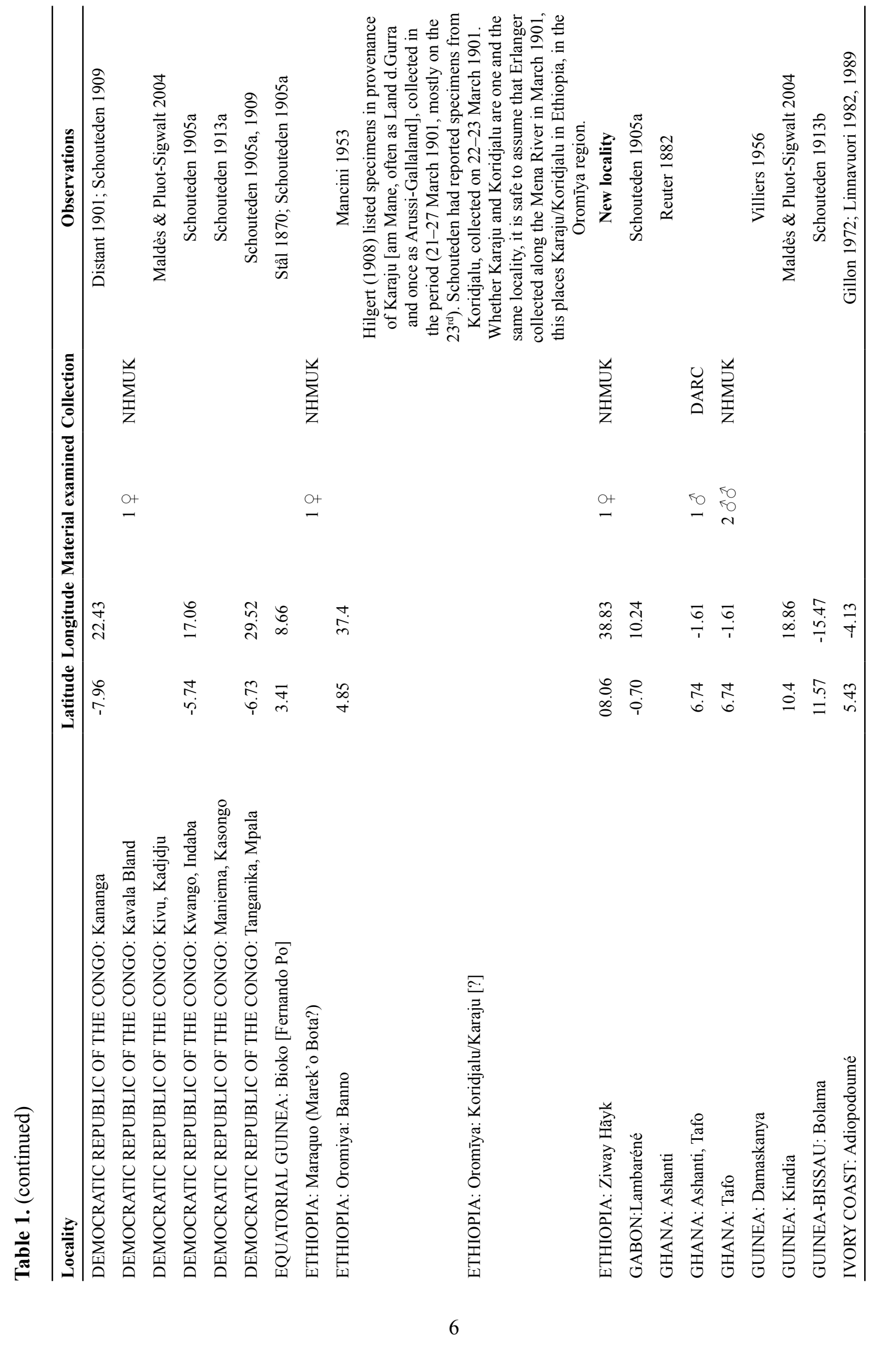




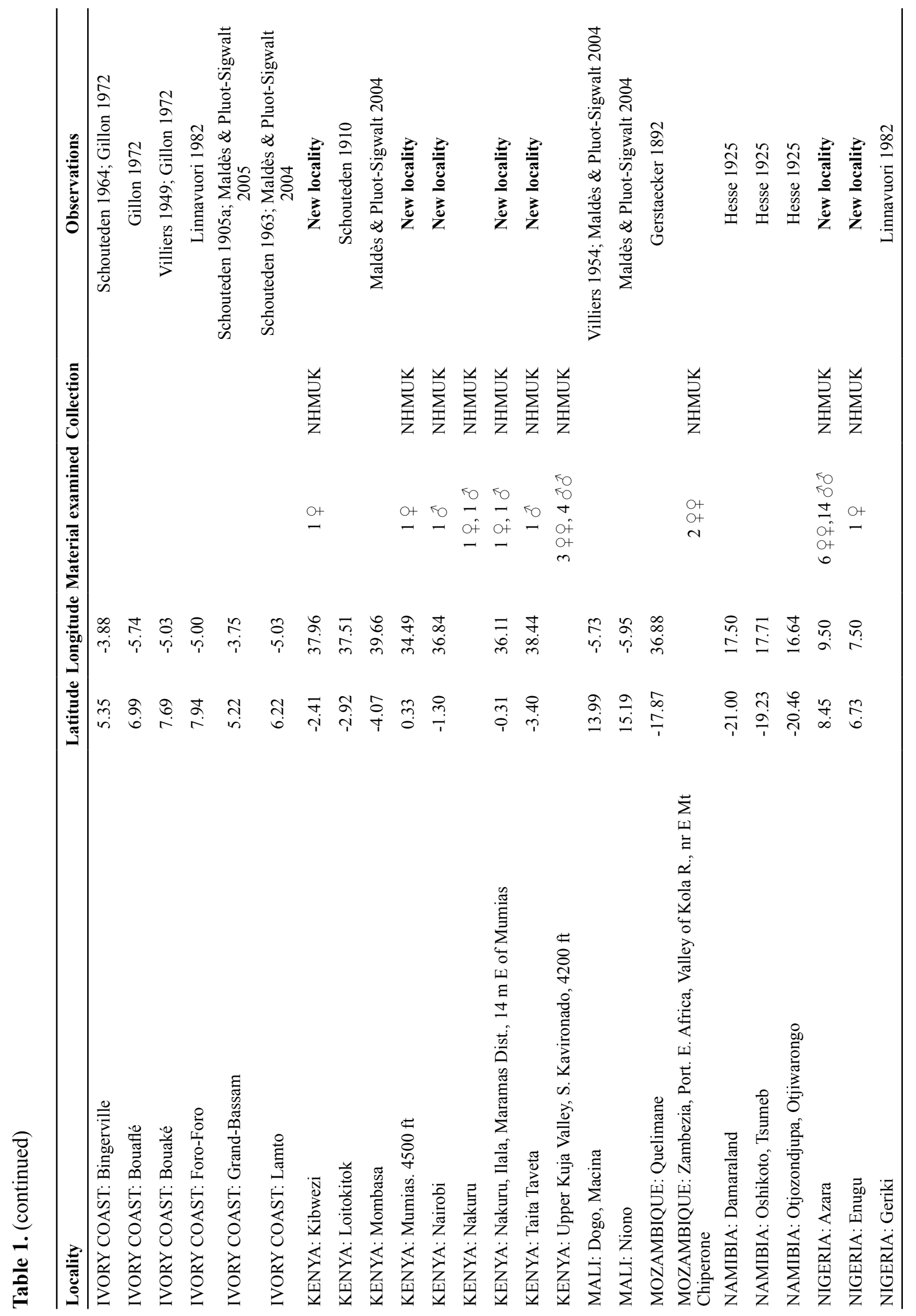




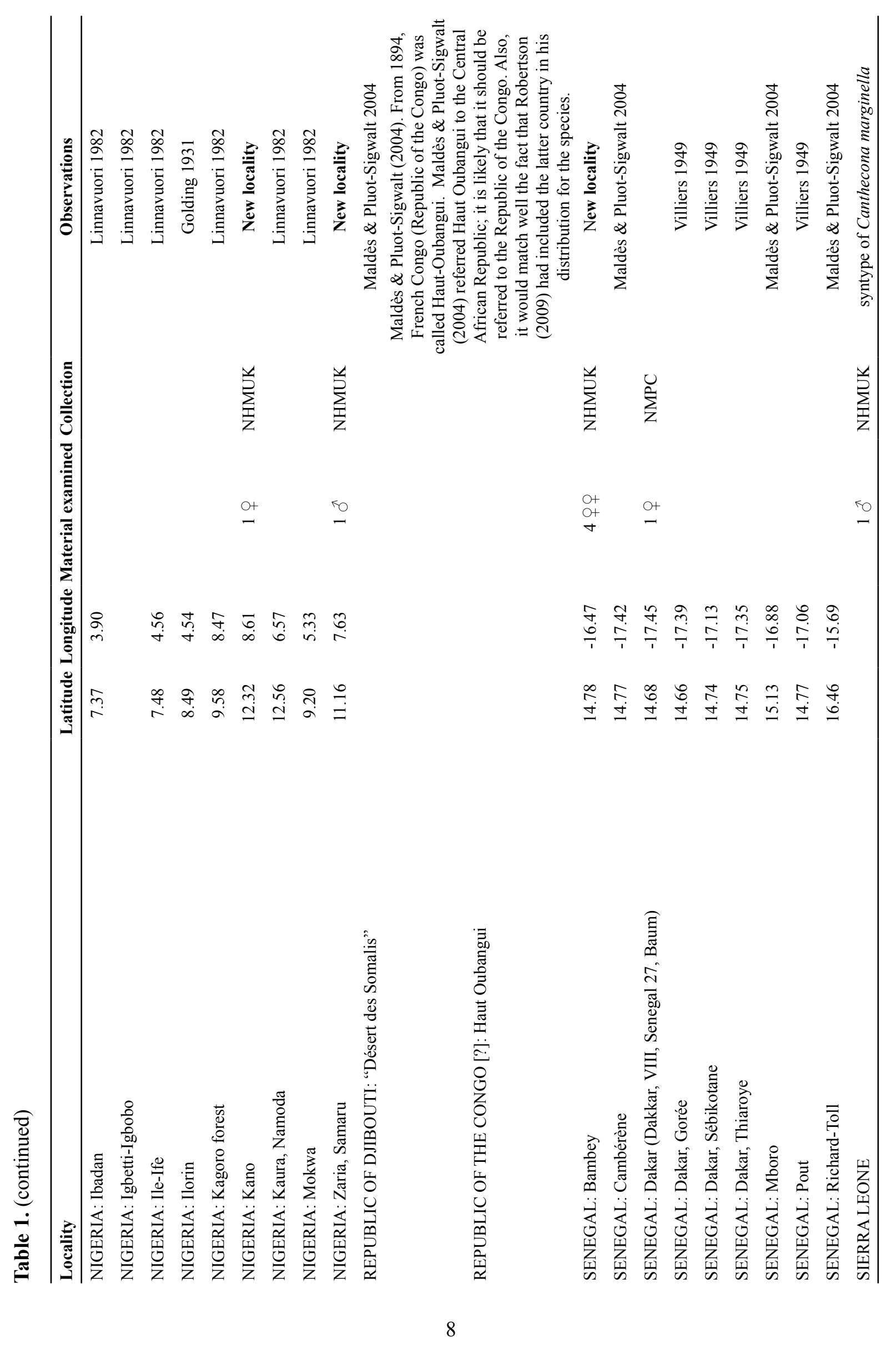




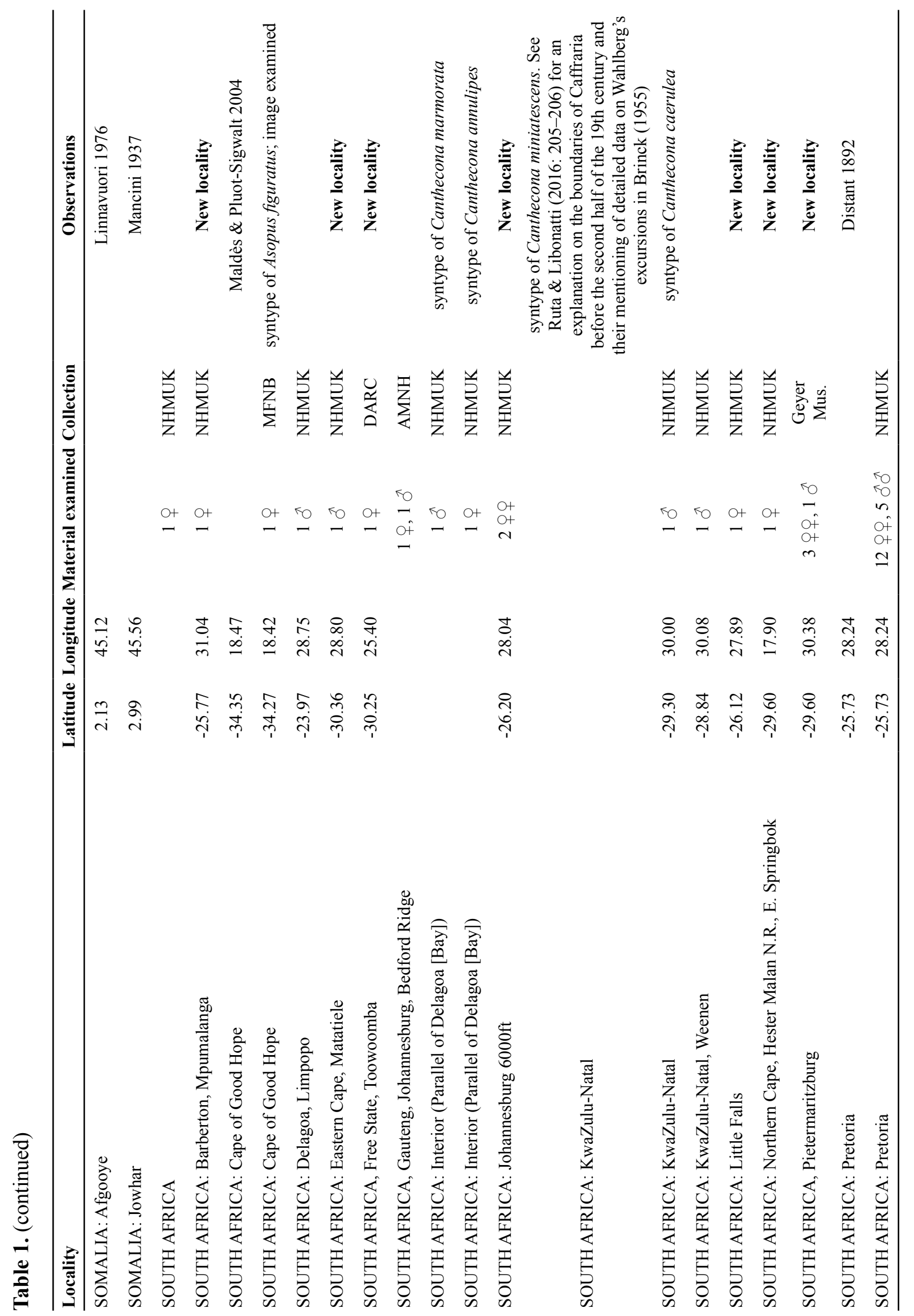




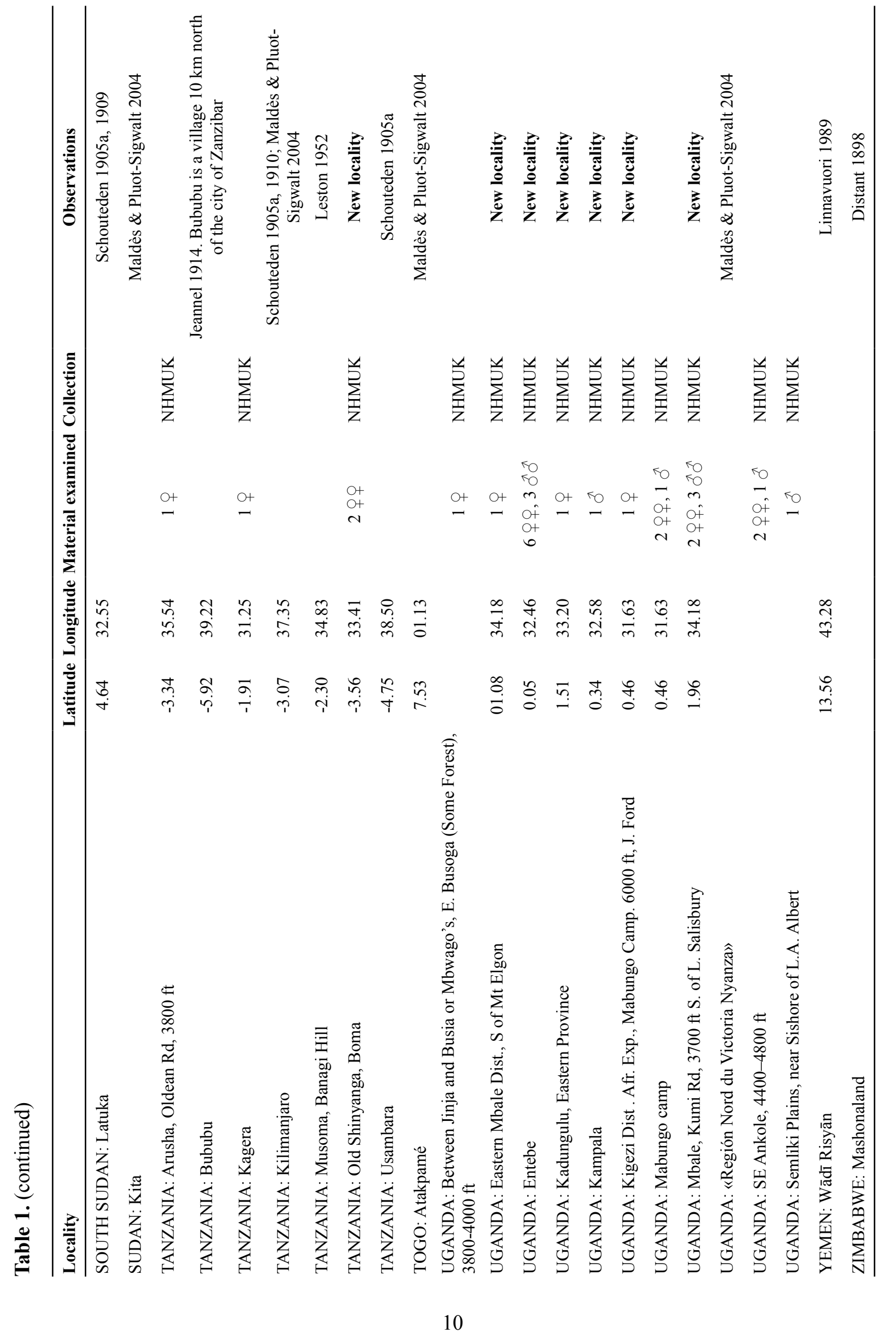




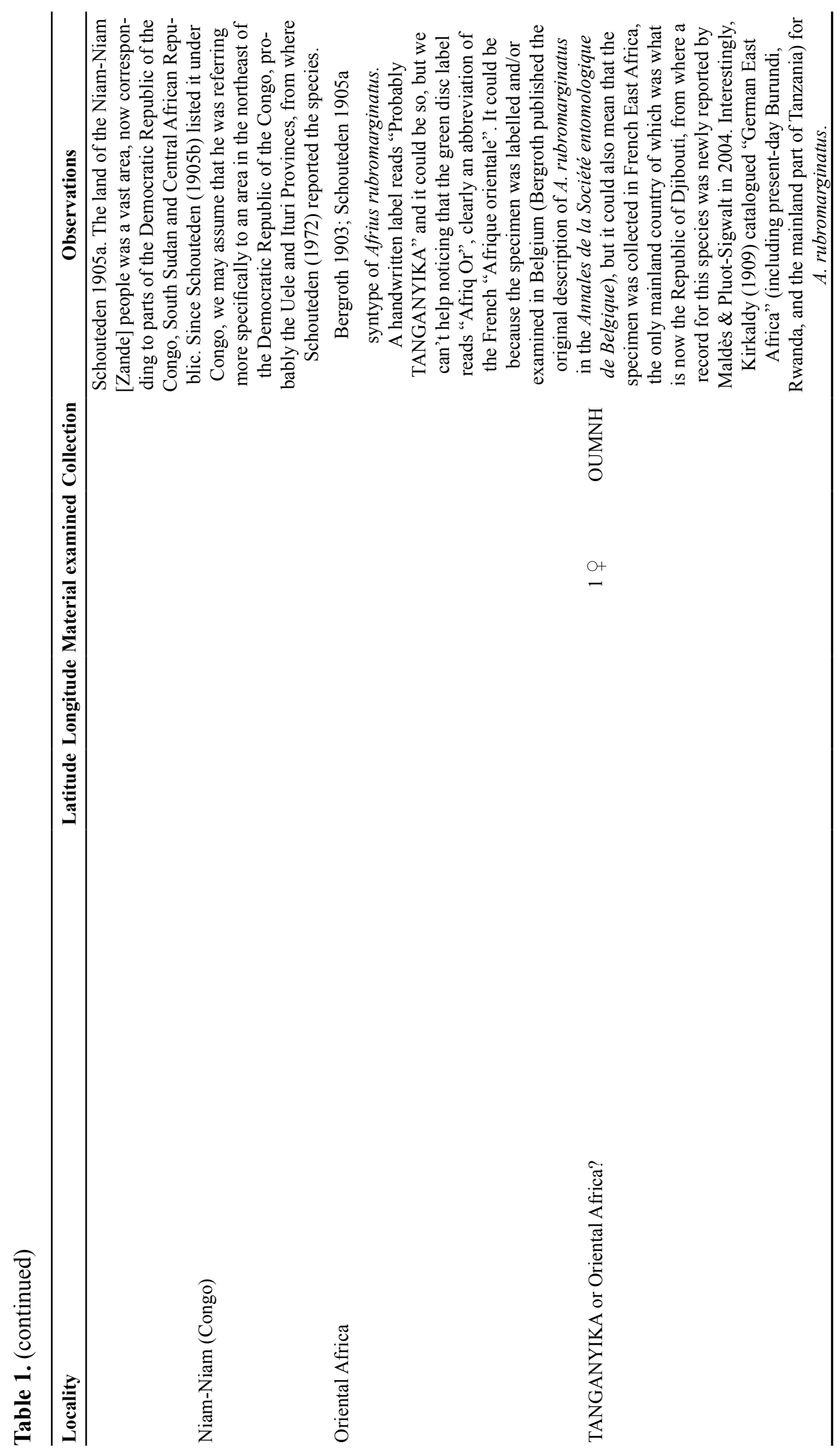


Table 2. Terminology of male genitalia from Singh-Pruthi 1925; Baker 1931; Dupuis 1955, 1970; Konstantinov \& Gapon 2005 and Gapon \& Konstantivov 2006.

\begin{tabular}{|c|c|c|c|c|}
\hline & Singh-Pruthi 1925 & Baker 1931 & $\begin{array}{c}\text { Dupuis 1955, } \\
1970\end{array}$ & $\begin{array}{l}\text { Konstantinov \& Gapon } 2005 \\
\text { Gapon \& Konstantivov } 2006\end{array}$ \\
\hline \multirow{5}{*}{$\begin{array}{l}\text { External } \\
\text { genitalia }\end{array}$} & segment IX & genital segment & $\begin{array}{l}\text { pygophore or } \\
\text { genital capsule }\end{array}$ & pygophore \\
\hline & - & $\begin{array}{l}\text { ventral and dorsal } \\
\text { borders }\end{array}$ & - & - \\
\hline & & genital plates & $\begin{array}{l}\text { processus } \\
\text { supérieurs }\end{array}$ & genital plates or parandria \\
\hline & segment $\mathrm{X}$ & proctiger & $\begin{array}{l}\text { anal tube or } \\
\text { proctiger }\end{array}$ & - \\
\hline & parameres & claspers & parameres & parameres \\
\hline \multirow{11}{*}{$\begin{array}{l}\text { Internal } \\
\text { genitalia }\end{array}$} & aedeagus & - & phallus & aedeagus \\
\hline & - & - & phalloteca & theca \\
\hline & - & - & - & basal theca \\
\hline & - & - & - & thecal shield \\
\hline & basal foramen & - & - & - \\
\hline & basal plates & - & - & - \\
\hline & vesica & - & vesica & $\begin{array}{l}\text { apical outgrowths of median } \\
\text { plates of the penis }+ \text { pons trans- } \\
\text { versus }+ \text { longitudinal filaments } \\
\text { of median plates of the penis }\end{array}$ \\
\hline & ejaculatory duct & & ductus seminis & seminal duct + vesica \\
\hline & gonophore & - & $\begin{array}{l}\text { secondary } \\
\text { gonopore }\end{array}$ & secondary gonopore \\
\hline & conjunctiva & - & conjunctiva & conjunctiva \\
\hline & ejaculatory reservoir & - & $\begin{array}{l}\text { ejaculatory } \\
\text { reservoir }\end{array}$ & ejaculatory reservoir \\
\hline
\end{tabular}

the labels or literature information had sufficient data. The map for the distributional records of species of Afrius (Fig. 1) was made using the ArcGIS Desktop program (ver. 10.4.1: http://desktop.arcgis.com). To understand how the old regions of Africa overlapped with current countries, we consulted the 'rare maps' website (https://www.raremaps.com).

Measurements in millimeters [mean \pm standard deviation (minimum and maximum values found among all specimens measured)] are given for the total length; length and width of the head, pronotum, scutellum and abdomen; and length of the antennal and labial segments. Genitalia were prepared with heated 10\% KOH aqueous solution. The terminology of Singh-Pruthi (1925), Baker (1931), Dupuis $(1955,1970)$, Konstantinov \& Gapon (2005) and Gapon \& Konstantinov (2006) was adopted for genitalic structures; a correspondence between different terms used for the male genitalia by these authors is given in Table 2 . We follow the terminology of Kment \& Vilímová (2010) for the external scent efferent system of the metathoracic gland. Drawings were carried out under a stereo microscope coupled with a camera lucida and edited with a vectorial image processor. 


\section{Results}

Order Hemiptera Linnaeus, 1758

Suborder Heteroptera Latreille, 1810

Family Pentatomidae Leach, 1815

Subfamily Asopinae Spinola, 1850

Afrius Stål, 1870

Cimex (Afrius) Stål, 1870: 44. Type species by subsequent designation (Schouteden 1907a: 51): Asopus figuratus Germar, 1838 (= Afrius purpureus (Westwood, 1837)).

Afrius - Lethierry \& Severin 1893: 214. — Schouteden 1907a: 50-52; 1909: 64; 1972: 106. — Kirkaldy 1909: 10. — Cachan 1952: 305. — Villiers 1952b: 81. — Mamet 1957: 34. — Gillon 1972: 351352. — Thomas 1994: 150-152. — Maldès \& Pluot-Sigwalt 2004: 20. — Rider 2006: 234. — Robertson 2009: 20-23.

\section{Diagnosis}

Lateral pronotal margins sinuous and crenulated on anterior half; frenal margin of scutellum longer than postfrenal part; abdominal basal tubercle short, not extending beyond metacoxae; profemur with a preapical spine; protibia very slightly expanded; male abdomen with or without ventral setose patches on segments V and VI; posterior angles of seventh abdominal segment obtuse (Fig. 8C, paVII); metapleural evaporatorium thinly surrounding peritreme.

\section{Redescription}

BODY LENGTH. 8.70-13.22 mm (우) and 9.00-11.00 (ふう).

BoDy. Oval or pentagonal, with variable colour patterns.

HEAD. Uniformly punctured, mandibular plates varying from equal to a little shorter or little longer than clypeus, with margins straight to slightly sinuous; ocelli placed close to an imaginary line connecting posterior margin of eyes; antenna with five antennomeres bearing thin setae, denser on fourth and fifth antennomeres; antennal tubercles partially visible from above, acute apically; bucculae rounded; labium robust, extending to posterior margin of metasternum.

Thorax. Pronotum hexagonal, uniformly punctured except on cicatrices; anterior margin concave; lateral margins sinuous, crenulated on anterior half; posterior angles with a prominent small spine; a thin central line without punctures forms a weak longitudinal medial carina that extends from anterior to posterior margin. Scutellum reaching an imaginary line connecting middle of each connexival segment V; a thin central line without punctures also forms a weak longitudinal medial carina, connected with a similar line on pronotum from anterior to posterior margins; frenal margins longer than postfrenal margins. Corium longer than scutellum, uniformly punctured, membrane surpassing apex of abdomen. Pro-, meso- and metasterna covered by small thin setae; prosternum lighter, with a weak median carina; mesosternum black between pro- and mesocoxae, slightly punctured, with central parallel horizontal stripes, and with a median light wide, rectangular and elevated carina, wider anteriorly; metasternum flat or slightly elevated. Metapleural evaporatorium narrowly surrounding peritreme, narrowly extending on posterior meso- and anterior metapleural margins, also extending to anterior angle of mesopleura. Peritreme disc: ostiolar opening laterally directed. Profemur with anteapical spine, protibia slightly expanded, meso- and metatibiae prismatic. 
ABDOMEN. Sparsely punctured, punctures less dense on disc, short basal tubercle anteriorly directed. Trichobothria aligned to an imaginary line connecting middle of spiracles. Posterolateral angles of abdominal sternites rounded.

\section{Male}

AbDomen. With or without ventral setose patches on segments V and VI.

Genitalia. Pygophore bowl-shaped, with setae on entire surface, denser between ventral border and inferior layer of ventral border, and on apex of posterolateral angles (Figs 3, 6, $10 \mathrm{~A}-\mathrm{F}$ ); dorsal border concave, weakly medially elevated (Figs 3, 6, $10 \mathrm{~A}, \mathrm{D}$, db); ventral border slightly concave (Figs 3, 6, 10, B, E, vb), medially emarginated in posterior view (Figs 3, 6, 10, C, F, vb), inferior layer slightly excavated (Figs 3, 6, 10, B-C, E-F, il). Posterolateral angles rounded, setose on apex (Figs 3, 6, 10, A-F, pa). Segment $X$ tubular, ventrally directed, dorsally sclerotized, medially carinated, setose, setae denser on apex (Figs 3, 6, 10, A, C-D, F, X). Genital plates between lateral walls of capsule and parameres (Figs 3, 6, 10, A, C-D, F, I, gp). Phallus. Phallotheca divided in a globose basal theca and a cup-like thecal shield (Figs $3 \mathrm{~J}-\mathrm{Q}, 6 \mathrm{~J}-\mathrm{R}, 10 \mathrm{~J}-\mathrm{R}$, ph, bt, ts). Ejaculatory reservoir contained inside basal theca (Figs $3 \mathrm{~J}-\mathrm{N}, 6 \mathrm{~J}-\mathrm{O}, 10 \mathrm{~J}-\mathrm{O}$, er). Basal foramen circular, reinforced by basal plates (Figs $3 \mathrm{~L}, \mathrm{~N}, 6 \mathrm{~L}, \mathrm{O}$, $10 \mathrm{~L}, \mathrm{O}, \mathrm{bf}, \mathrm{bp}$ ). Vesica partially inserted in phallotheca, golf club-shaped in lateral view, bearing two elongated filaments and a central elevated portion with microsculptures (Figs $3 \mathrm{~J}-\mathrm{Q}, 6 \mathrm{~J}-\mathrm{R}, 10 \mathrm{~J}-\mathrm{R}, \mathrm{v}$ ); ductus seminis running between filaments of vesica, ending on a secondary gonopore, dorsally directed (Figs 3J-Q, 6J-R, 10J-R, ds, sg). Conjunctival lobes paired, posteriorly directed, globose, with apices endowed with a set of small sculptured processes (Figs 3J-P, 6J-P, 10J-Q, cl, sp).

\section{Female}

Genitalia. Gonocoxites VIII subtriangular, posterior margins sinuous, sutural margins straight, juxtaposed, setae on posterior and sutural margins (Figs 4, 7, 11, A-B, gcVIII). Laterotergites VIII triangular, longer than wide, with spiracles on basal angle (Figs 4, 7, 11, A-B, laVIII). Exposed portion of gonocoxites IX rectangular, wider than long, slightly covering proximal lateral margins of laterotergites IX (Figs 4, 7, 11, A-B, gcIX). Exposed portion of laterotergites IX digitiform, setose on apex, not attaining band uniting laterotergites VIII, separated from each other by gonocoxites IX and segment X (Figs 4, 7, 11, A-B, laIX). Segment X trapezoidal (Figs 4, 7, 11, A-B, X). Inner portion of gonocoxites IX projected in $1+1$ straight elongated arms, variable in extension, and with apices rounded or acute (Figs 4, 7, 11, C-D, gcIX). Gonapophyses IX with 1+1 variable secondary thickenings (Figs 4, 7, 11, C-D, gpIX). Ring sclerites absent. Thickening of vaginal intima elongated (Figs 4, 7, 11, C-D, vi). Pars intermedialis small (Figs 4, 7, 11, C-D, pi), narrower than median duct of vesicular area. Capsula seminalis oval, longer and wider than pars intermedialis (Figs 4, 7, 11, C-D, cs).

\section{Distribution}

Throughout Africa and adjacent islands (Fig. 1).

\section{Remarks}

Afrius was created as a subgenus of Cimex Linnaeus, 1758 by Stål (1870), but not within the present understanding of Cimex, since Cimex currently corresponds to a genus of Cimicidae. At the time of the description, three species of Afrius were recognized, viz. Cimex (Afrius) figuratus (Germar, 1838), C. (Afrius) purpureus (Westwood, 1837) and C. (Afrius) flavirostris Stål, 1864, while two others (Canthecona marmorata Dallas, 1851 and C. annulipes Dallas, 1851) were mentioned as species incertae sedis. Lethierry \& Severin (1893) included all the above five species in Afrius. Schouteden (1907a) divided the genus into two subgenera (Afrius s. str. and Subafrius Schouteden, 1907a), separating them by the size of the scutellum and by the presence of abdominal silky patches in the male of the subgenus Afrius. 
Afrius can be differentiated from most African genera of Asopinae by the following combined characteristics: lateral pronotal margins crenulated on anterior half, presence of a well-developed spine on profemora, and abdominal basal tubercle short, not extending beyond metacoxae. The genus shares these features only with Canthecona Amyot \& Serville, 1843, Glypsus Dallas, 1851 and Picromerus Amyot \& Serville, 1843; however, the posterior abdominal segment of Canthecona is acuminate, not obtuse as in Afrius; the abdominal tubercle is bifid in Glypsus, not single as in Afrius; and the metapleural evaporatorium is more developed in Picromerus in comparison with Afrius.

We maintain the division of Afrius in two subgenera based on the presence or absence of abdominal glandular patches (Schouteden 1907a; Thomas 1994) and morphological differences of the male genitalia, described below. We do not consider, however, these subgenera as two distinct genera because the presence or absence of abdominal glandular patches can be interspecifically variable in other genera of Asopinae, as in Macrorhaphis Dallas, 1851 (Thomas 1994). Besides, the species of Afrius present many similarities of general morphology, of female genitalia, and of the pygophore, mainly the genital plates. Perhaps a phylogenetic study may better elucidate the classification of Afrius in future.

\section{Key to the species of Afrius Stål, 1870}

1. Scutellum wider than long, humeral pronotal angles laterally well projected to acute angle (Fig. 2B, D, F, H, J). Male abdomen without setose patches on segments V and VI (Fig. 2C, L), parameres with two evident rami (Fig. 3G-H)

A. (Subafrius) flavirostrum (Signoret, 1861)

- Scutellum longer than wide (Figs 5B, D, 8B, D, F, H, J, L, N, P), humeral pronotal angles slightly projected to acute (Figs 5B, 9B), rounded (Fig. 9A, C) or triangular angle (Fig. 9D). Male abdomen with setose patches on segments $\mathrm{V}$ and VI (Fig. 8C), parameres without two evident rami, triangular (Figs 6G-H, 10G-H)

2. Postfrenal lobe of scutellum enlarged, constriction line (sc) broader than adjacent region (ac) of corium until radial vein (Fig. 8H). Humeral angles slightly emarginated (Fig. 9) …………………………………………...... A. (Afrius) purpureus (Westwood, 1837)

- Postfrenal lobe narrow, constriction line (sc) equal or shorter than adjacent region (ac) of corium until radial vein (Fig. 5D). Humeral angles not emarginated (Fig. 5B, D)

A. (Afrius) kolleri Schouteden, 1911

Afrius (Subafrius) Schouteden, 1907

Afrius (Subafrius) Schouteden, 1907a: 51. Type species by original monotypy: Picromerus flavirostrum Signoret, 1861.

Afrius (Subafrius) - Kirkaldy 1909: 10. — Cachan 1952: 306. — Thomas 1994: 152. — Maldès \& Pluot-Sigwalt 2004: 20. — Rider 2006: 234.

\section{Diagnosis}

Males without abdominal glandular patches; parameres divided in two lobes; dorsal disc of vesica covered by fine and inconspicuous microsculptures.

Afrius (Subafrius) flavirostrum (Signoret, 1861)

Figs $1-4$

Picromerus flavirostrum Signoret, 1861: 921.

Cantheconidea migratoria Distant, 1913: 144-145. Syn. nov.

Afrius williamsi Miller, 1952: 183-184. Syn. nov. 
Canthecona flavirostris (incorrect subsequent spelling) - Stål 1864: 68.

Cimex (Afrius) flavirostris (incorrect subsequent spelling) - Stål 1870: 44.

Afrius flavirostris (incorrect subsequent spelling) - Lethierry \& Severin 1893: 214 [with "Stål 1864" as authority]. - Schouteden 1905a: 151-153.

Afrius (Subafrius) flavirostrum - Schouteden 1907a: 51. - Kirkaldy 1909: 10. — Cachan 1952: 306. — Thomas 1994: 152. — Maldès \& Pluot-Sigwalt 2004: 20. — Gapon \& Konstantinov 2006: 809.

Afrius williamsi - Williams 1951: 461. — Jolivet \& Théodoridès 1953: 5. — Orian 1956: 642. — Mamet 1957: 35. - Cox 1996: 38.

Subafrius flavirostrum - Orian 1965: 116.

Afrius (Subafrius) migratorius - Thomas 1994: 152.

Afrius (Subafrius) williamsi - Thomas 1994: 152.

Afrius flavirostrum - Kuklinski \& Borgemeister 2002: 59.

\section{Types examined}

MADAGASCAR - Picromerus flavirostrum Signoret, 1861; syntype đ̇; labels: "Madagasc Coll. Signoret.", "flavirostr det. Signoret.", "flavirostrum d. Schouteden."; Fig. 2A-C; NHMW • syntype o; labels: "Madagascar. Coll. Signoret.", "flavirostrum", "flavirostrum d. Schouteden.", "flavirostr. det. Signoret.", "Afrius flavirostrum Type Sign."; Fig. 2E-F; NHMW • syntype $O$; labels: "Madag.", "Stål”, “Type”, "Typus", "NHRS-GULI 000057896”; Fig. 2D; NHRS-GULI 000057896. These three syntypes were examined by photos.

SEYCHELLES ISLANDS - Cantheconidea migratoria Distant, 1913; syntype +; labels: bluemargined syntype disc label; red-margined type disc label "Aldabra. APT. 1907", "Percy Sladen Trust Expedition. 1911-497.", "Canthecona migratoria type Dist.", "NHMUK 010592166"; Fig. 2G-H; NHMUK010592166.

MAURITIUS - Afrius williamsi Miller, 1952; holotype ổ; labels: red-margined holotype disc label, "MAURITIUS. Coll. J.R. Williams i.1949.", “182”, "Pres by Com Inst Ent BM 1950 - 262”, “Afrius williamsi sp.n det. N.C.E. Miller. 1950.”, "COM INST ENT. COLL. NO. 11607”, "Predaceous on Schematiza cordiae, Barb", "NHMUK 010592172"; Fig. 2I-J; NHMUK 010592172 • paratype +; labels: yellow-margined paratype disc label, "MAURITIUS Reduit. xii.1948 Coll. J.R. Williams", "182", "Pres by Com Inst Ent BM 1950 - 262", "Afrius williamsi sp.n det. N.C.E. Miller. 1950.", "COM INST. ENT. COLL. NO. 11607", "Predaceous on Schematiza cordiae, Barb", "NHMUK 010592173"; Fig. 2K-L; NHMUK 010592173 • paratype \%; labels: yellow-margined paratype disc label, "MAURITIUS Reduit. xii.1948 Coll. J.R. Williams", "182", "Pres by Com Inst Ent BM 1950 - 262", “Afrius williamsi sp.n det. N.C.E. Miller. 1950.", “COM INST. ENT. COLL. NO. 11607”,

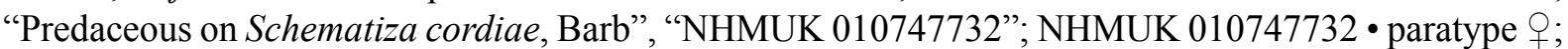
labels: yellow-margined paratype disc label, "MAURITIUS Reduit. I.1949 Coll. J.R. Williams", "182", "Pres by Com Inst Ent BM 1950 - 262", "Afrius williamsi sp.n det. N.C.E. Miller. 1950.", "COM INST. ENT. COLL. NO. 11607", "Predaceous on Schematiza cordiae, Barb", "NHMUK 010747733"; NHMUK 010747733 - paratype $\bigcirc$; labels: yellow-margined paratype disc label, "MAURITIUS Reduit. vi.1949 Coll. J.R. Williams", "182", "Pres by Com Inst Ent BM 1950 - 262", "Afrius williamsi sp.n det. N.C.E. Miller. 1950.", "COM. INST. ENT. COLL. NO. 11607”, "Predaceous on Schematiza cordiae, Barb", "NHMUK 010747734"; NHMUK 010747734 • paratype +; labels: yellow-margined paratype disc label, "Dept. of Agric. MAURITIUS", "Pres by Com. Inst. Ent. B.M.1948-38", "COM. INST. ENT. COLL. NO. 10958”, “182”, "NHMUK 010747735”; NHMUK 010747735 • paratype ô;; labels: yellow-margined paratype disc label, "MAURITIUS Reduit. i.1949 Coll. J.R. Williams", "182", "Pres by Com Inst Ent BM 1950 - 262", "Afrius williamsi sp.n. det. N.C.E. Miller. 1950.", "COM INST. ENT. COLL. NO. 11607", "Predaceous on Schematiza cordiae, Barb", "NHMUK 010747736"; NHMUK 010747736 • paratype ${ }^{\top}$; labels: yellow-margined paratype disc label, "MAURITIUS Reduit 
A. Moutia II.v.1948”, “Pres by Com. Inst. Ent. B.M.1948-38”, “COM. INST. ENT. COLL. NO. 10958”, "Preying on Schematiza cordiae", “182”, "NHMUK 010747737”; NHMUK 010747737 • paratype +; labels: yellow-margined paratype disc label, "MAURITIUS Reduit. i.1949 Coll. J.R. Williams", "Afrius williamsi sp.n. det. N.C.E. Miller. 1950.", "Press by Com Inst Ent B M 1950 - 262", "COM INST. ENT. COLL. NO. 11607”, “182", "Predaceous on Schematiza cordiae, Barb”, "NHMUK 010747738”; NHMUK 010747738 • paratype + ; labels: yellow-margined paratype disc label, "MAURITIUS Reduit. i.1949 Coll. J.R. Williams", "Afrius williamsi sp.n. det. N.C.E. Miller. 1950.”, "Press by Com Inst Ent B M 1950 - 262"; "182", "Predaceous on Schematiza cordiae, Barb", "NHMUK 010747739"; NHMUK 010747739 • paratype $\$$; labels: yellow-margined paratype disc label, "Dept. of Agric. MAURITIUS Reduit 19.IV.1948", "J.R. Williams", "Pres by Com. Inst. Ent. B.M.1948-38”, “COM. INST. ENT. COLL. NO. 10958”, “182”, “NHMUK 010747740”; NHMUK 010747740.

\section{Diagnosis}

Scutellum wider than long, humeral pronotal angles laterally well projected to an acute angle; male abdomen without setose patches; parameres with two distinct lobes.

\section{Redescription}

BoDy. Elongated oval, yellowish to brown, usually with a pale posterior margin of the scutellum.

HEAD. Subrectangular, wider than long, uniformly punctured; mandibular plates equal or slightly shorter than and twice as wide as clypeus, anteriorly rounded, with margin straight to slightly sinuous; antennomeres yellow to brownish, black coloured on apical halves of third to fifth antennomeres, bearing thin setae, denser on third to fifth antennomeres; proportion of lengths of antennomeres: II $\geq \mathrm{IV}>\mathrm{V}>$ III $>$ I; labium robust, reaching metasternum, last segment darker than preceding; proportion of lengths of labiomeres: II $>$ I $\geq$ III $>$ IV.

THORAX. Pronotum hexagonal, uniformly punctured except on cicatrices, twice or more as wide as long, cicatrices flat. Anterior margin concave. Lateral margins sinuous, slightly crenulated on anterior half, humeral angle laterally projected, emarginated, apices acute. Scutellum wider than long. Corium longer than scutellum, reaching connexival segment VI, uniformly punctured, membrane surpassing apex of abdomen (Fig. 2).

Male abdomen. Without setose patches; parameres biramous (Fig. 3F-H, par).

\section{Male}

MEASUREMENTS $(\mathrm{n}=5)$. Head length $1.82 \pm 0.18(1.68-2.10)$; width $1.93 \pm 0.15(1.87-2.13)$; pronotum length $2.52 \pm 0.48$ (1.85-3.22); width $6.44 \pm 0.64$ (5.64-7.25); scutellum length $3.02 \pm 0.32$ (2.66-3.48); width $3.22 \pm 0.34$ (2.88-3.75); lengths of antennomeres: I $0.32 \pm 0.05$ (0.27-0.37), II $1.26 \pm 0.11$ (1.21.39), III $1.12 \pm 0.23$ (0.97-1.37), IV $1.32 \pm 0.21$ (1.12-1.54); V $1.16 \pm 0.13$ (1.05-1.31); lengths of labiomeres: I $0.94 \pm 0.02$ (0.93-0.97), II $1.13 \pm 0.09$ (1.05-1.23), III $0.97 \pm 0.10$ (0.90-1.90), IV 0.81 \pm 0.02 (0.78-0.82); length of abdomen $4.50 \pm 0.70$ (4.00-5.00); width $4.82 \pm 0.25$ (4.65-5.00); total length $9.87 \pm 0.71(9.19-11.00)$.

Genitalia. Genital plates elongated and cylindrical (Fig. 3A, C-D, F, I, gp). Parameres long, biramous, head V-shaped divided into one process long, acute and another shorter, rounded, dorsally directed, extended beyond pygophore (Fig. 3A-H, par). Phallus. Thecal shield about twice as long as basal theca, widely opened posteriorly (Fig. 3J-Q, ts). Vesica subrectangular in dorsal view (Fig. 3O-Q, v), golf club-shaped in lateral view, with apex subtriangular and a central, slightly elevated, rounded portion covered by fine and inconspicuous microsculptures (Fig. 3L-N, v, m); ductus seminis enlarged near 


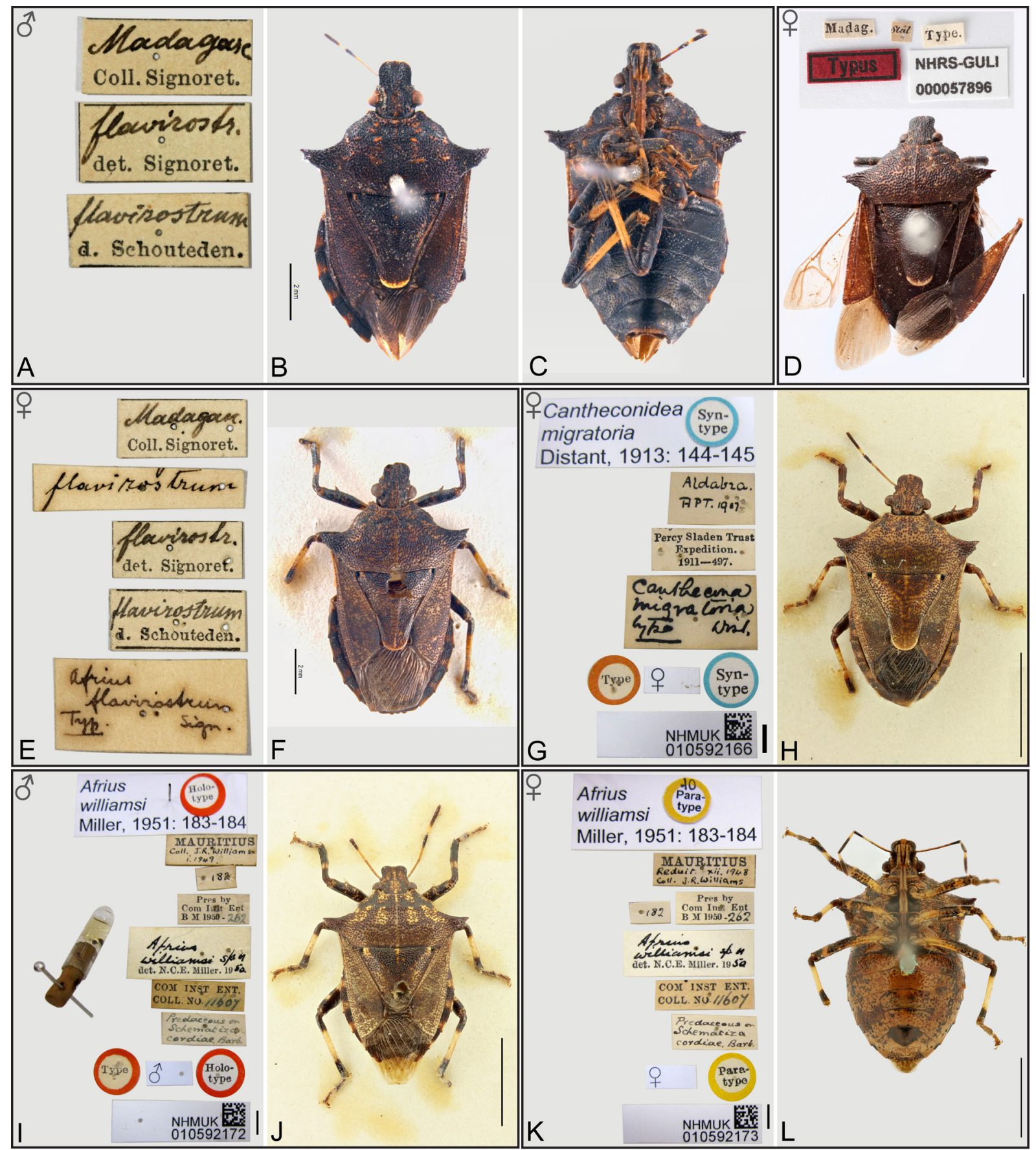

Fig. 2. Afrius (Subafrius) flavirostrum (Signoret, 1861), type specimens. A-C. Picromerus flavirostrum Signoret, 1861, syntype $\widehat{\partial}$, labels, dorsal and ventral habitus, respectively (images courtesy of Herbert Zettel and Harald Bruckner, NHMW). D. Picromerus flavirostrum, syntype $q$, labels and dorsal habitus (image courtesy of Gunvi Lindberg, NHRS). E-F. Picromerus flavirostrum, syntype $q$, labels and dorsal habitus (images courtesy of Herbert Zettel and Harald Bruckner, NHMW). G-H. Cantheconidea migratoria Distant, 1913, syntype ${ }^{+}$, labels and dorsal habitus (NHMUK). I-J. Afrius williamsi Miller, 1951, holotype $\widehat{\text { ô }}$, labels and dorsal habitus (NHMUK). K-L. Afrius williamsi, paratype ${ }_{+}$, labels and ventral habitus (NHMUK). Scale bars: $4 \mathrm{~mm}$. 

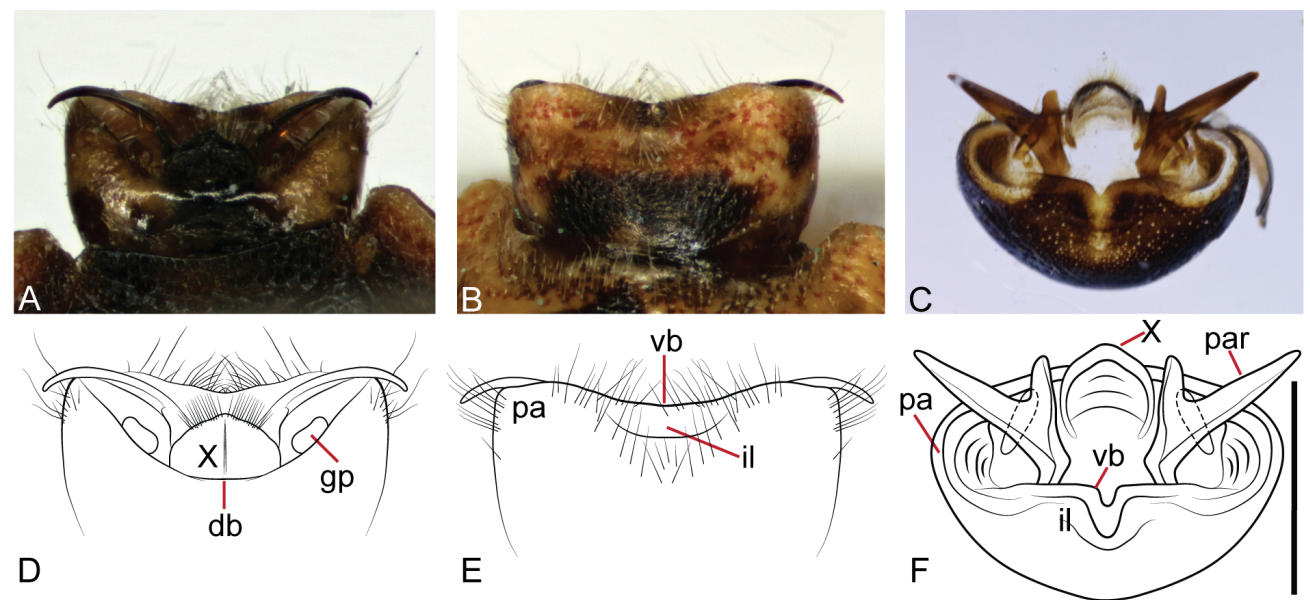

$\mathrm{D}$

$\mathrm{E}$
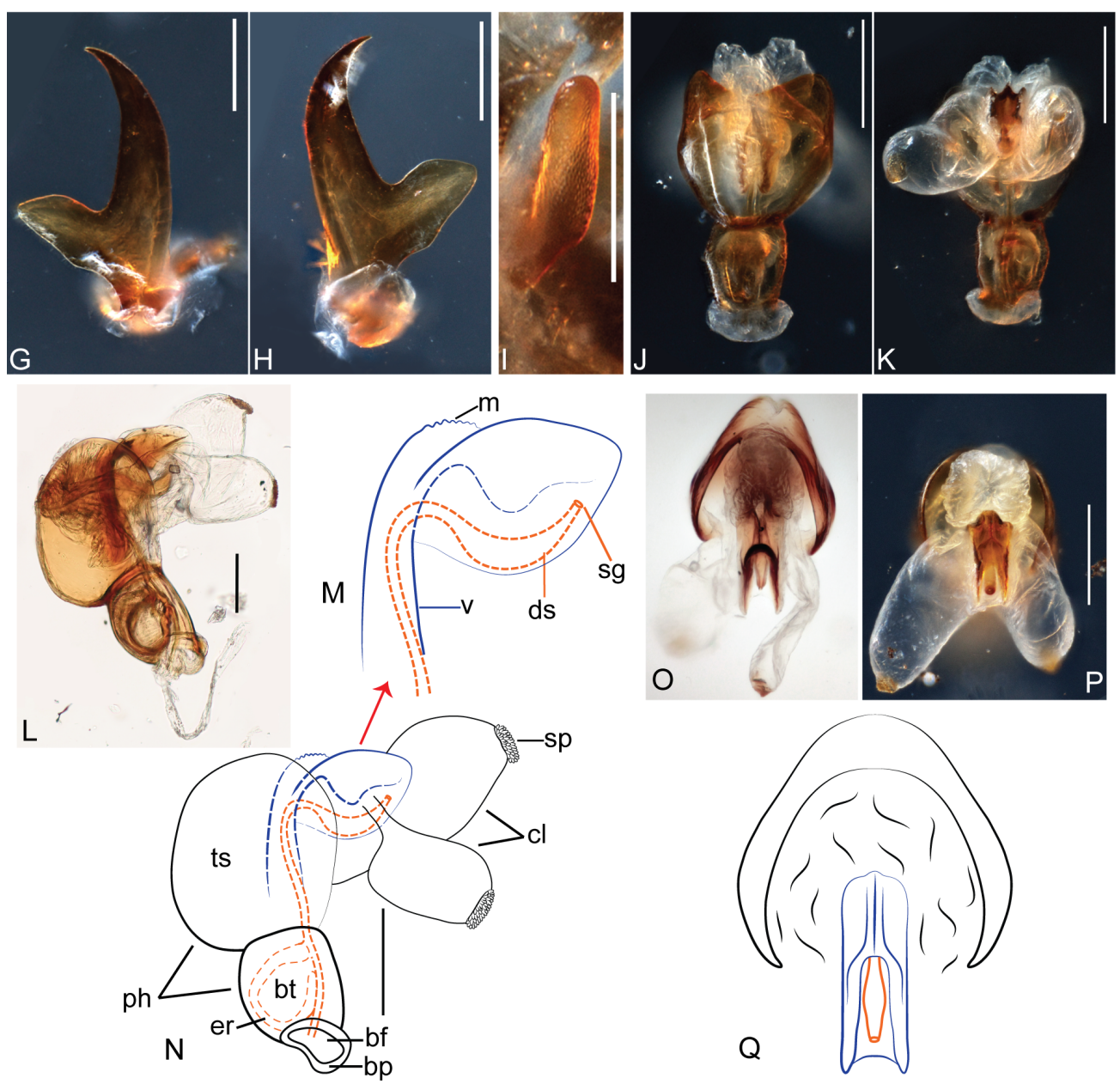

Fig. 3. Afrius (Subafrius) flavirostrum (Signoret, 1861), male genitalia. A-F. Pygophore in dorsal (A, D), ventral (B, E) and posterior (C, F) views. G-H. Right paramere in lateral views, internal and external respectively. I. Right genital plate, dorso-posterior view. J-L. Phallus in anterior, posterior and lateral views, respectively. M. Detail of the vesica in lateral view, also represented in N. N. Drawing of the phallus. $\mathbf{O}-\mathbf{Q}$. Phallus in dorsal view. Abbreviations: $b f=$ basal foramen; $b p=$ basal plates; $b t=$ basal theca; $\mathrm{cl}=$ conjunctival lobes; $\mathrm{db}=$ dorsal border; $\mathrm{ds}=$ ductus seminis; $\mathrm{er}=$ ejaculatory reservoir; $\mathrm{gp}=$ genital plates; $\mathrm{il}=$ inferior layer; $\mathrm{m}=$ microsculptures; $\mathrm{pa}=$ posterolateral angles; par = parameres; $\mathrm{ph}=$ phallotheca; $\mathrm{sg}=$ secondary gonophore; $\mathrm{sp}=$ conjunctival process; $\mathrm{ts}=$ thecal shield; $\mathrm{v}=$ vesica; $\mathrm{vb}=$ ventral border; $\mathrm{X}=$ segment $\mathrm{X}$. Scale bars: $\mathrm{F}=0.5 \mathrm{~mm} ; \mathrm{G}-\mathrm{Q}=0.25 \mathrm{~mm}$. 
apex (Fig. 3K-Q, ds). Apices of conjunctival lobes globose, endowed with a set of sculptured processes, forming a subrectangular projection in lateral view (Fig. 3K-P, cl, sp).

\section{Female}

MEASUREMENTS $(\mathrm{n}=5)$. Head length $1.87 \pm 0.10(1.80-1.95)$; width $1.78 \pm 0.18(1.65-1.91)$; pronotum length $2.62 \pm 0.17$ (2.5-2.74); width $3.48 \pm 0.07$ (3.43-3.53); scutellum length $3.01 \pm 0.07$ (2.96-3.07); width $3.24 \pm 0.18$ (3.11-3.37); length of antennomeres: I $0.30 \pm 0.0(0.30-0.30)$, II $1.29 \pm 0.03$ (1.27$1.31)$, III $1.08 \pm 0.0(1.08-1.08)$, IV $1.28 \pm 0.05$ (1.24-1.31); V $1.16 \pm 0.06$ (1.12-1.20); length of labiomeres: I $0.97 \pm 0.06$ (0.93-1.01), II $1.14 \pm 0.03$ (1.12-1.16), III $0.94 \pm 0.0$ (0.94-0.94), IV $0.86 \pm$
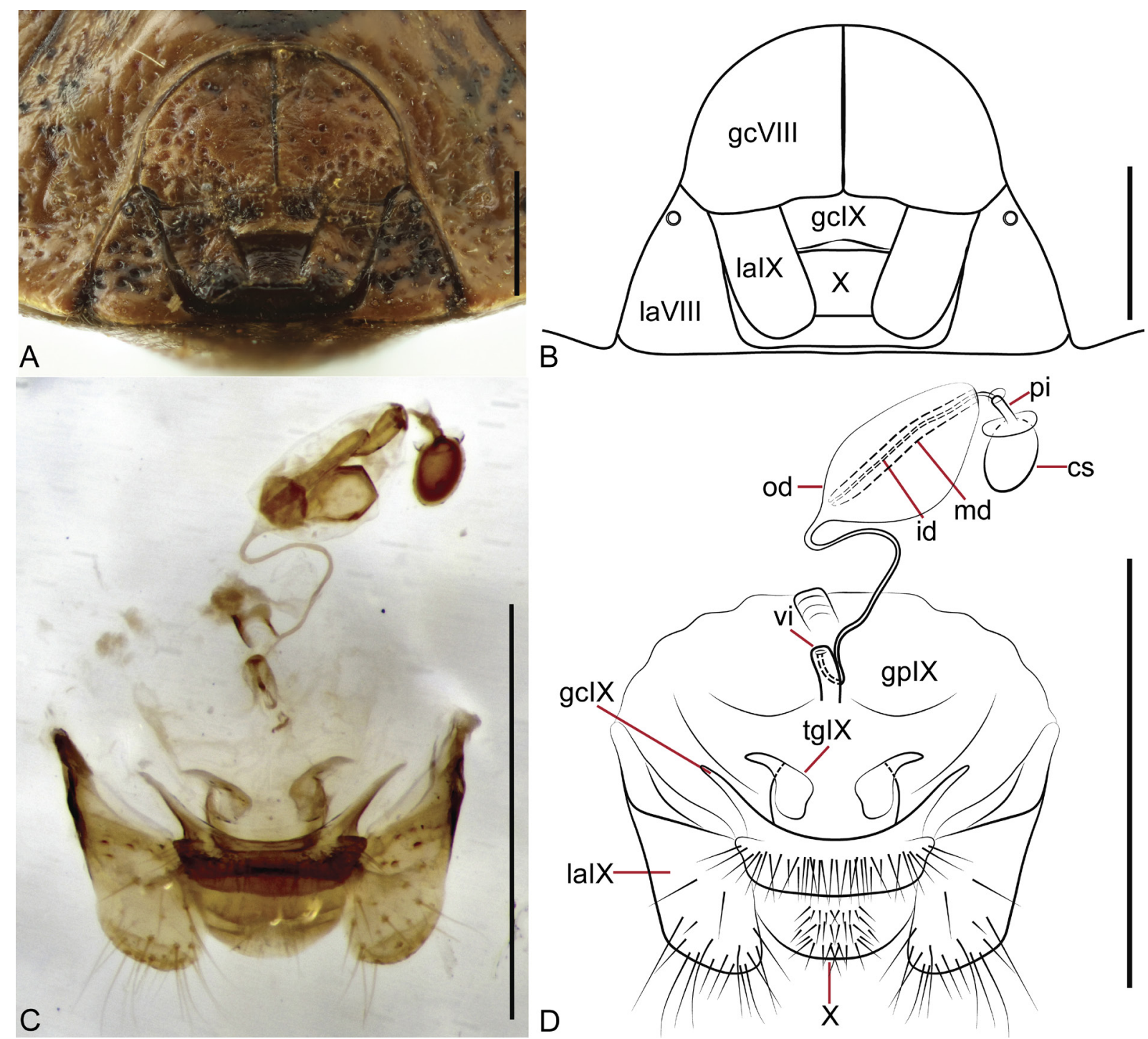

Fig. 4. Afrius (Subafrius) flavirostrum (Signoret, 1861), female genitalia. A-B. Genital plates, ventroposterior view. C-D. Inner genitalia, ventral view. Abbreviations: cs = capsula seminalis; gcVIII = gonocoxites VIII; gcIX = gonocoxites IX; gpIX = gonapophyses IX; id = inner duct; laVIII = laterotergites VIII; laIX = laterotergites IX; md = median duct of vesicular area; od = outer duct of vesicular area; $\mathrm{pi}=$ pars intermedialis; $\operatorname{tgIX}=$ secondary thickening of gonapophyses IX; $\mathrm{vi}=$ thickening of vaginal intima; $X=$ segment $X$. Scale bars: $A-B=1 \mathrm{~mm} ; C-D=0.5 \mathrm{~mm}$. 
ROELL T. et al., Revision of the African shieldbug genus Afrius

$0.06(0.82-0.90)$; length of abdomen $5.12 \pm 0.05$ (5.08-5.16); width $5.24 \pm 0.0$ (5.24-5.24); total length $9.51 \pm 1.14(8.70-10.32)$.

Genitalia. Gonocoxites VIII slightly longer than wide, mesial portions of posterior margins slightly constricted (Fig. 4A-B, gcVIII). Median and inner ducts of vesicular area with uniform diameter (Fig. 4C-D, md, id).

\section{Distribution}

Madagascar (Signoret 1861), Seychelles Islands (Distant 1913), Mauritius (Miller 1952) (Table 1, Fig. 1).

\section{Remarks}

Although this species seems similar to A. (Afrius) purpureus (Westwood, 1837) in general aspect, this is the most distinct species of the genus: the scutellum is wider than long, the males do not have abdominal glandular patches, the parameres are divided into two arms, and the vesica is shorter and less microsculptured in comparison to that of A. (Afrius) purpureus and A. (Afrius) kolleri Schouteden, 1911. Because of the aforementioned, we have kept the subgeneric classification, grouping $A$. kolleri and A. purpureus in the subgenus Afrius, and A. flavirostrum in the subgenus Subafrius.

When describing A. williamsi, Miller (1952) compared his new species with A. marmoratus (Dallas, 1851), but did not mention A. (Subafrius) flavirostrum.

The two new synonymies proposed herein were previously pointed out by Orian in his unpublished thesis (Orian 1965).

Afrius (Afrius) Stål, 1870

Cimex (Afrius) Stål, 1870: 44.

Afrius (Afrius) - Schouteden 1907a: 51-52. — Kirkaldy 1909: 10. — Thomas 1994: 151-152. — Maldès \& Pluot-Sigwalt 2004: 20.

\section{Diagnosis}

Males with abdominal glandular patches; parameres not divided in two lobes; dorsal disc of vesica covered by dense and demarcated microsculptures.

Afrius (Afrius) kolleri Schouteden, 1911

Figs 1, 5-7

Afrius kolleri Schouteden, 1911: 180; lectotype herein designated.

Afrius kolleri - Schouteden 1963: 399; 1972: 106. — Gillon 1972: 352; 1974: 219. — Thomas 1994: 151. — Maldès \& Pluot-Sigwalt 2004: 20. — Robertson 2009: 22-23.

\section{Types examined}

DEMOCRATIC REPUBLIC OF THE CONGO • Afrius kolleri Schouteden, 1911; lectotype $\widehat{\delta}$ (here designated); labels: "Holotypus", "MUSÉE DU CONGO Galli-Koko Kasai R. CARLIER", "Afrius Stål kolleri Schout.", "Afrius kolleri n. sp.”; Fig. 5A-B; RMCA • Paralectotype O; labels: "Paratypus", "MUSÉE DU CONGO LUKOMBE. 6.X.08 A. Koller”; Fig. 5C-D; RMCA. 


\section{Diagnosis}

Scutellum longer than wide, postfrenal lobe narrow; humeral pronotal angles not emarginated; male abdomen with setose patches on segments V and VI, parameres triangular, without two distinct lobes.

\section{Redescription}

BoDy. Pentagonal elongated, reddish to brown, usually with black stripes on head, pronotum, scutellum, and corium.

HEAD. Quadrate, as long as wide or slightly wider than long, uniformly punctured; mandibular plates equal or slightly longer than clypeus, and slightly wider than clypeus, with margins straight to slightly sinuous; clypeus black; ocelli surrounded by subquadrate black spots; antennomeres usually black, proportion of lengths of antennomeres: V $>$ IV $>$ III $=$ II $>$ I; labium robust, reaching metasternum; proportion of lengths of labiomeres: II $>$ I $>$ III $>$ IV.

THorax. Pronotum hexagonal, uniformly punctured, wider than long, with 1+1 black transversal stripes on cicatrices, $1+1$ black vertical spots on humeral angles and 3 longitudinal stripes on disc; cicatrices flat, black, sometimes with a central red spot, demarcated by punctures; anterior margin concave; lateral margins sinuous, crenulated on anterior half; humeri triangular, not emarginated. Scutellum longer than wide, uniformly punctured, reaching an imaginary line connecting middle of connexival segments $\mathrm{V}$, partially or entirely emarginated in black and with one triangular black central spot along frenal lobe; postfrenal lobe narrow, narrower than corium at same region. Corium longer than scutellum, reaching connexival segment VI. Protibiae slightly expanded (Fig. 5).

MaLE ABdomen. With setose patches on segments V and VI; parameres uniramous, triangular.

\section{Male}

Measurements $(\mathrm{n}=3)$. Head length $1.95 \pm 0.20(1.72-2.07)$; width $2.07 \pm 0.06(2.02-2.15)$; pronotum length $2.76 \pm 0.23$ (2.52-2.98); width $4.86 \pm 0.40$ (4.53-5.32); scutellum length $3.23 \pm 0.13$ (3.08$3.33)$; width $2.90 \pm 0.09$ (2.80-2.96); length of antennomeres: I $0.31 \pm 0.05(0.27-0.37)$, II $1.05 \pm 0.07$ (0.97-1.12), III $1.08 \pm 0.04$ (1.05-1.12), IV $1.34 \pm 0.06$ (1.30-1.38); V $1.50 \pm 0.00$ (1.50-1.50); length of labiomeres: I $0.86 \pm 0.03(0.84-0.90)$, II $1.16 \pm 0.03$ (1.12-1.18), III $0.92 \pm 0.02(0.90-0.93)$, IV
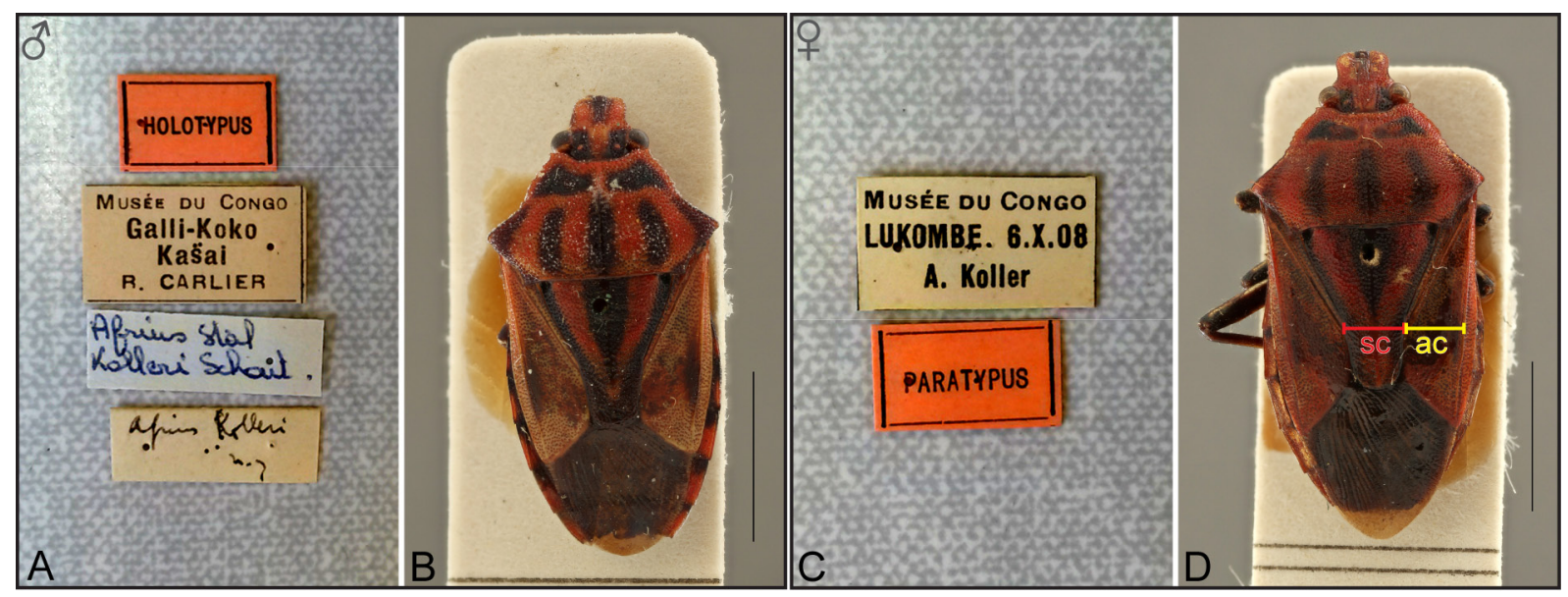

Fig. 5. Afrius (Afrius) kolleri Schouteden, 1911, types. A-B. Lectotype $\widehat{\sigma}$, labels and dorsal habitus (RMCA). C-D. Paralectotype 9 , labels and dorsal habitus (RMCA). Abbreviations: ac $=$ corium adjacent to the scutellum constriction; $s c=$ scutellum constriction. Scale bars: $4 \mathrm{~mm}$. 

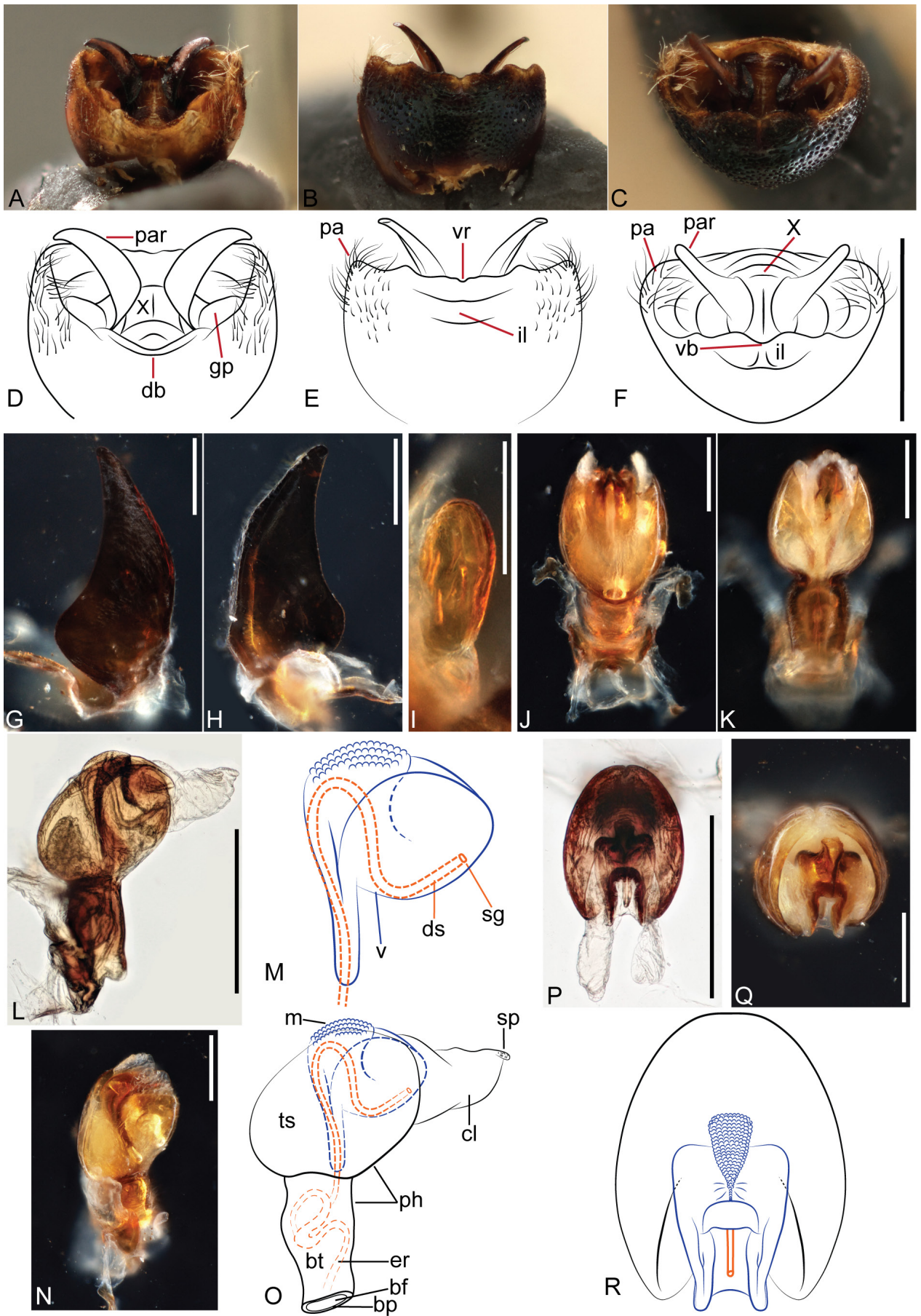

Fig. 6. Afrius (Afrius) kolleri Schouteden, 1911, male genitalia. A-F. Pygophore in dorsal (A, D), ventral $(\mathrm{B}, \mathrm{E})$ and posterior $(\mathrm{C}, \mathrm{F})$ views. G-H. Right paramere in lateral views, internal and external respectively. I. Right genital plate, dorso-posterior view. J-L. Phallus in anterior, posterior and lateral views, respectively. M. Detail of the vesica in lateral view, also represented in O. N. Phallus in dorsolateral view. O. Drawing of the phallus. P-R. Phallus in dorsal view. Abbreviations: $b f=$ basal foramen; $\mathrm{bp}=$ basal plates; $\mathrm{bt}=$ basal theca; $\mathrm{cl}=$ conjunctival lobes; $\mathrm{db}=$ dorsal border; $\mathrm{ds}=$ ductus seminis; $\mathrm{er}=$ ejaculatory reservoir; $\mathrm{g} p=$ genital plates; $\mathrm{il}=$ inferior layer; $\mathrm{m}=$ microsculptures; $\mathrm{pa}=$ posterolateral angles; $\mathrm{par}=$ parameres; $\mathrm{ph}=$ phallotheca; $\mathrm{sg}=$ secondary gonophore; $\mathrm{sp}=$ conjunctival process; $\mathrm{ts}=$ thecal shield; $v=$ vesica; $v b=$ ventral border; $X=$ segment $X$. Scale bars: $D-F, L, P=0.5 \mathrm{~mm} ; \mathrm{G}-\mathrm{K}, \mathrm{N}$, $\mathrm{Q}=0.25 \mathrm{~mm}$. 
$0.92 \pm 0.02$ (0.90-0.93); length of abdomen $5.12 \pm 0.17$ (5.00-5.24); width $4.56 \pm 0.15$ (4.45-4.67); total length $10.11 \pm 1.00(9.00-10.97)$.

Genitalia. Genital plates cylindrical (Fig. 6A, D, I, gp). Parameres long, uniramous, head triangularly elongated, dorsally directed, extended beyond pygophore (Fig. 6A-H, par). Phallus. Thecal shield and basal theca subequal in length (Fig. 6L, N-O, ts, bt). Vesica shield-shaped in dorsal view (Fig. 6P-R), golf club-shaped in lateral view (Fig. 6L-O, v), with apex rounded and a central elevated microsculptured
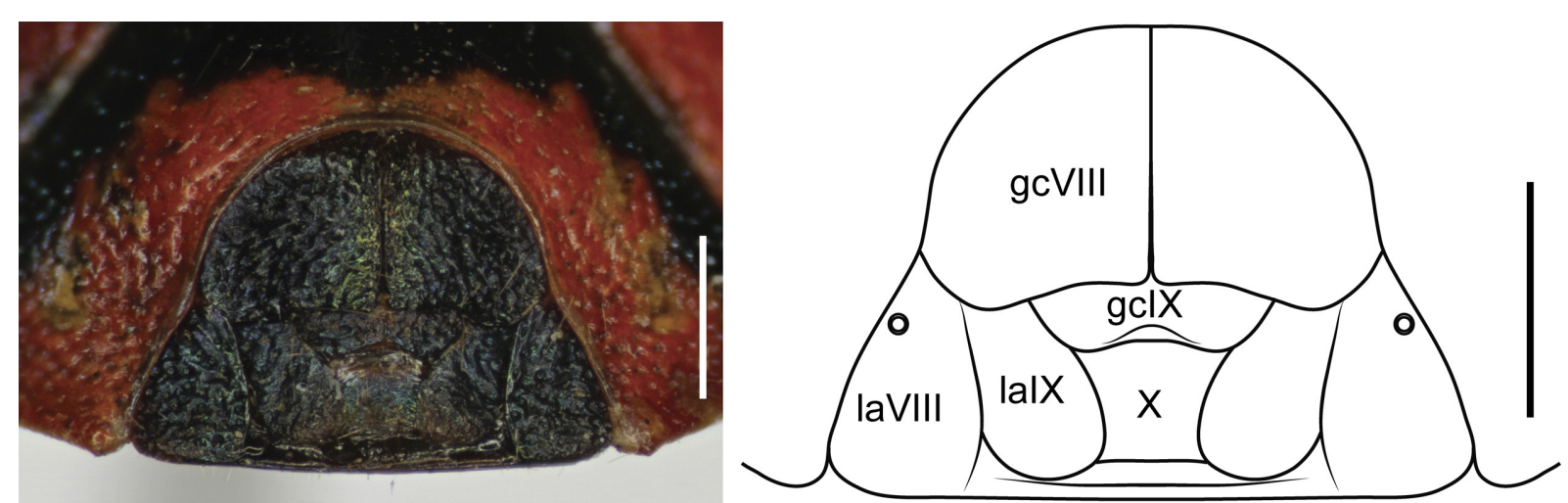

A

$\mathrm{B}$
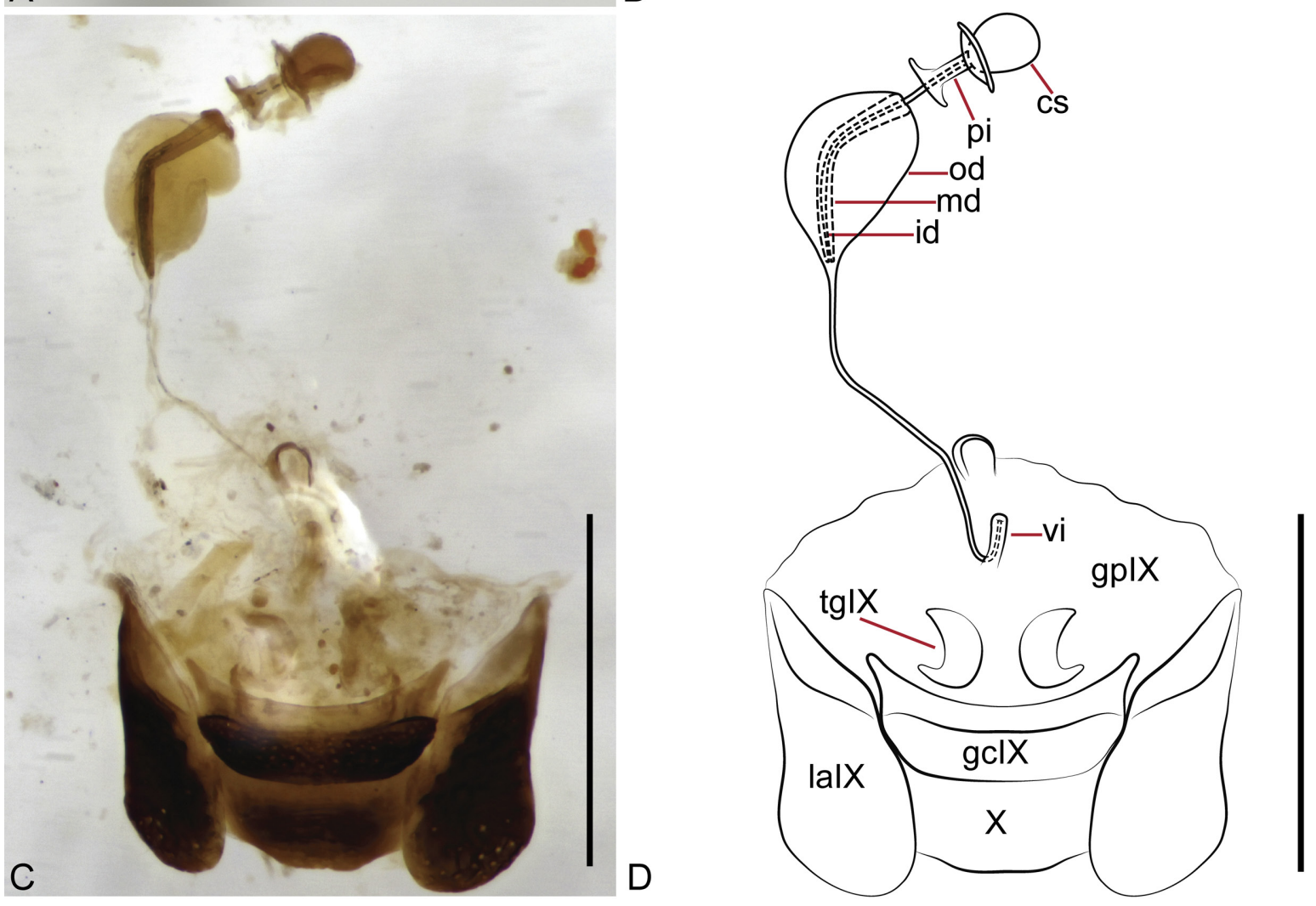

Fig. 7. Afrius (Afrius) kolleri Schouteden, 1911, female genitalia. A-B. Genital plates, ventroposterior view. C-D. Inner genitalia, ventral view. Abbreviations: $\mathrm{cs}=$ capsula seminalis; gcVIII = gonocoxites VIII; gcIX = gonocoxites IX; gpIX = gonapophyses IX; laVIII = laterotergites VIII; id = inner duct; laIX = laterotergites IX; $\mathrm{md}=$ median duct of vesicular area; od $=$ outer duct of vesicular area; $\mathrm{pi}=$ pars intermedialis; $\operatorname{tgIX}=$ secondary thickening of gonapophyses IX; vi $=$ thickening of vaginal intima; $\mathrm{X}=$ segment X. Scale bars: A-B $=1 \mathrm{~mm} ; \mathrm{C}-\mathrm{D}=0.5 \mathrm{~mm}$. 
portion (Fig. 6L-O, m), this central portion rounded in lateral view, subtriangular in dorsal view; ductus seminis with uniform diameter (Fig. 6L-R, ds). Apices of conjunctival lobes with inconspicuous sculptured process (Fig. 6L, O-P, sp).

\section{Female}

Measurements $(\mathrm{n}=5)$. Head length $2.20 \pm 0.09$ (2.06-2.30); width $2.20 \pm 0.12$ (2.05-2.35); pronotum length $3.33 \pm 0.24$ (3.06-3.63); width $5.81 \pm 0.31$ (5.56-6.03); scutellum length $3.94 \pm 0.27$ (3.714.30); width $3.34 \pm 0.26$ (3.07-3.75); length of antennomeres: I $0.35 \pm 0.01(0.33-0.37)$, II $1.18 \pm 0.07$ (1.12-1.25), III $1.18 \pm 0.08$ (1.12-1.27), IV $1.47 \pm 0.13$ (1.31-1.62); V $1.42 \pm 0.0$ (1.42-1.42); length of labiomeres: I $1.04 \pm 0.13(0.90-1.23)$, II $1.27 \pm 0.07$ (1.20-1.35), III $1.00 \pm 0.08$ (0.93-1.12), IV 0.98 \pm 0.10 (0.83-1.08); length of abdomen $6.40 \pm 0.45$ (6.03-7.09); width $5.59 \pm 0.34$ (5.40-6.20); total length $11.98 \pm 0.86(11.12-13.22)$.

Genitalia. Gonocoxites VIII slightly longer than wide, posterior margins sinuous (Fig. 7 A-B, gcVIII). Median and inner ducts of vesicular area slightly widening to apex (Fig. 7 C-D, md, id).

\section{Distribution}

Democratic Republic of the Congo (Schouteden 1911), Ivory Coast (Gillon 1972), Ethiopia, Uganda (Thomas 1994), Cameroon (Maldès \& Pluot-Sigwalt 2004) (Table 1, Fig. 1).

\section{Remarks}

This species is apparently most similar to A. (Afrius) purpureus because they both present glandular patches on the abdominal venter of males, parameres that are not divided in two rami and vesica microsculptures.

The description of Afrius kolleri was based on an unspecified number of specimens of both sexes (Schouteden 1911). We examined two specimens at RMCA: a male labelled as holotype and a female labelled as paratype. Since no holotype was designated in the original description, both of them must be considered as syntypes; accordingly, we hereby designate the male specimen as lectotype.

\section{Afrius (Afrius) purpureus (Westwood, 1837)}

Figs $1,8-11$

Pentatoma yolofa Guérin-Méneville, 1831: 55, fig. 2 and legend (syn. Stål 1870: 44).

Pentatoma purpurea Westwood, 1837: 43.

Asopus figuratus Germar, 1838: 185-186 (syn. Schouteden 1905: 147, as a var. of Canthecona purpurea).

Canthecona caerulea Dallas, 1851: 89 (syn. Stål 1862: 496, as var. of Canthecona Yolofa Guérin; syn. Stål 1870: 44).

Canthecona marginella Dallas, 1851: 89 (syn. Stål 1862: 496, as var. of Canthecona Yolofa Guérin; syn. Stål 1870: 44).

Canthecona marmorata Dallas, 1851: 90 (synonymy with Canthecona purpurea suspected by Schouteden 1905a: 150). syn. nov.

Canthecona annulipes Dallas, 1851: 90-91 (syn. Schouteden 1905a: 149-150, with Canthecona marmorata; synonymy with Canthecona purpurea suspected by Schouteden 1905a: 150). syn. nov.

Canthecona miniatescens Stål, 1854: 213 (syn. Stål 1864: 66-67, with Canthecona figurata, as var. b.; syn. Kirkaldy 1909: 10, with Afrius purpureus, as a var.).

Afrius rubromarginatus Bergroth, 1903: 289. syn. nov.

Pentatoma (Eurydema) yolofa - Laporte 1833: 61.

Canthecona Yolofa - Amyot \& Serville 1843: 82. — Dallas 1851: 89. — Stål 1864: 67-68. — Larousse 1890: 724 [without any capital letters].

Cimex (Pentatoma) yolofa - Guérin-Méneville 1844: 344 [description] 
Asopus figuratus - Herrich-Schaffer 1844: 113, fig. 710.

Canthecona figurata - Stål 1864: 66-67. — Gerstaecker 1892: 345.

Cimex (Afrius) figuratus - Stål 1870: 44.

Cimex (Afrius) purpureus - Stål 1870: 44. — Distant 1884: 459.

Cimex marmoratus - Stål 1870: 46.

Cimex annulipes - Stål 1870: 46.

Canthecona Ylofa (incorrect subsequent spelling) - Wallengren 1875: 133.

Afrius purpureus - Reuter 1882: 9. - Lethierry \& Severin 1893: 214. — Schouteden 1907a: 51-52; 1909: 64; 1910: 91; 1913a: 190; 1913b: 324; 1963: 399; 1964: 95; 1972: 106. — Kirkaldy 1909: 10. — Vuillet \& Vuillet 1911: 277; 1912: 445. — Hollrung 1912: 280. — Jeannel 1913: 97. — Zacher 1921: 139. - Lehmann 1922: 129. - Hesse 1925: 39. - Carpenter 1926: liii. - Golding 1931: 222. — Van Heerden 1931: 131, fig. cvii. — Hargreaves 1937: 518. - Villiers 1949: 88; 1952a: 1211; 1952b: 82; 1954: 230; 1956: 213. - Risbec 1950: 126, 397, 447, 457. — Cachan 1952: 305; 1965: 5-32. — Leston 1952: 895. — Mancini 1953: 202. — Lindberg 1958: 31. — Le Pelley 1959: 166. - Caswell 1962: 26. — Girard 1969: 7, 52. - Herting 1971: 80; 1976: 71. — Gillon 1972: 351-352; 1974: 219, 241, 246, 251, 266, 270, 285, 287. — Linnavuori 1975: 124; 1976: 129; 1982: 164. — Medler 1980: 123. — Bourdouxhe \& Jolivet 1981: 46-48. — Nuamah 1982: 11. — Nonveiller 1984: 54, 142. — Balsbaugh 1988: 276. — Couilloud 1989: 207-208. - Sylvie et al. 1989: 277. — Quicke et al. 1992: 1024. — Van Harten 1993: 247. — Bijlmakers \& Verhoek 1995: 147, 317. - Schaefer 1996: 41. — Jolivet 1997: 153. - Boulard 1998: 41. — Dolling et al. 1999: 73. - Beenen \& Hawkeswood 2004: 473. - Tchibozo \& Braet 2004: 161. - Arechavaleta et al. 2005: 76. - Renou 2007: 30. — Poutouli et al. 2011: 9, 54-56, 58, 76. — Agboton et al. 2014: 9. —Crétenet \& Gourlot 2015: 138.

Afrius purpureus var. marginella - Reuter 1882: 9. - Kirkaldy 1909: 10.

Cimex (Afrius) purpureus var. marginella - Distant 1884: 459.

Cimex purpureus - Distant 1890: LIII [with Hope as author, not Westwood].

Cimex figuratus - Distant 1892: 248 [description and illustration of unnamed variety].

Afrius ? annulipes - Lethierry \& Severin 1893: 214. http://www.palaeontos.be/13/det13.html

Afrius figuratus - Lethierry \& Severin 1893: 214. — Distant 1898: 308; 1901: 27. — Howard 1906: 731.

— Leston 1954: 680 (and in title). — Maitai 1958: 291. — Le Pelley 1959: 257; 1968: 195, 501.

— McDonald 1966: 44. — Cobben 1968: 116. - Herting 1973: 84, 85, 87. - Medler 1980: 123.

— Smith \& Barfield 1982: 263. — Scholtz \& Holm 1985: 147. — Merrett 1986: 549. — Matanmi \& Hassan 1987: 376. — Fry 1989: 108. — Mugo 1995: 2099. — Schaefer 1996: 44. — Sileshi et al. 2000: 41, 48.

Afrius ? marmoratus - Lethierry \& Severin 1893: 214.

Canthecona purpurea - Schouteden 1905a: 146; 1905b: 15.

Canthecona purpurea var. figuratus - Schouteden 1905a: 147.

Canthecona marmorata - Schouteden 1905a: 149-150.

Canthecona rubromarginata - Schouteden 1905a: 150-151.

Afrius marmoratus - Schouteden 1907a: 51. — Kirkaldy 1909: 10. — Thomas 1994: 151. — Robertson 2009: 22.

Afrius rubromarginatus - Schouteden 1907a: 52. — Bergroth 1908: 182. — Kirkaldy 1909: 10. Thomas 1994: 151. — Maldès \& Pluot-Sigwalt 2004: 20. — Robertson 2009: 22. — Krüger \& Deckert 2016: 46.

Afrius purpureus var. figuratus - Schouteden 1907a: 51-52; 1909: 64. — Mancini 1937: 43. — Le Pelley 1959: 54.

Afrius purpureus var. caerulea - Kirkaldy 1909: 10.

Afrius purpureus var. figurata - Kirkaldy 1909: 10.

Afrius purpureus var. miniatescens - Kirkaldy 1909: 10.

Afrius yolofus - Dupuis 1952: 454. — Leston 1954: 680 (in a note). — Thomas 1994: 151. — Maldès \& Pluot-Sigwalt 2004: 20. — Rebagliati et al. 2005: 201. — Kerisew 2011: 91. 
Afrius purpureus purpureus - Linnavuori 1989: 13.

Afrius purpureus figuratus - Linnavuori 1989: 12-13 [with unnamed variety].

Afrius yolofa - Sileshi et al. 2001: 289; 2004: 6, 18. — Kerzhner et al. 2004: 18. — Rider 2006: 234. -

Robertson 2009: 21-22. — Matesco et al. 2014: 352.

\section{Types examined}

AFRICA - Pentatoma purpurea Westwood, 1837; syntype + ; labels: "Type Hem: 242 PENTATOMA PURPUREA WESTWOOD HOPE DEPT. OXFORD", "TYPE = WEST. (HOPE) C. Hemipt. 1837 Part. I. page 43 Distant, P.Z.S., 1900, p. 807-825.”, “Africa”, “Afrius purpureus Westw.”; Fig. 8E-F; OUMNH.

"EAST AFRICA" • Afrius rubromarginatus Bergroth, 1903; syntype +; labels: "Type Hem: 704 AFRIUS RUBROMARGINATUS BERGROTH. HOPE DEPT. OXFORD", "Afrius rubro=marginatus Bergr.", "Probably TANGANYIKA", "Ann. Soc. Ent. Belg. 47: 289", "Afriq Or", “3/”, "TYPE”; Fig. 8O-P; OUMNH.

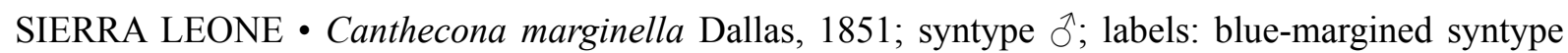
disc label, red-margined type disc label, "87a", "Canthecona marginella identified by Dallas", "a", "NHMUK 010592170"; Fig. 8I-J; NHMUK 010592170.

SOUTH AFRICA • Asopus figuratus Germar, 1838; syntype $\odot$; labels: "figuratus Germ. Promont. b. sp. Collect. Germ.”, “Afrius figuratus (Germ)”, "7968”, "Typus”; Fig. 8G-H; MFNB.

SOUTH AFRICA • Canthecona caerulea Dallas, 1851; syntype ${ }^{\uparrow}$; labels: blue-margined syntype disc label, red-margined type disc label, "40 626 329", "Canthecona caerulea identified by Dallas", "a", "NHMUK 010592171”; Fig. 8A-C; NHMUK 010592171.

SOUTH AFRICA - Canthecona marmorata Dallas, 1851; syntype ${ }^{3}$; labels: blue-margined syntype disc label, red-margined type disc label, "Int. S. Africa / 4319", "3. Canthecona marmorata,", "a", "NHMUK 010592164"; Fig. 8K-L; NHMUK 010592164.

SOUTH AFRICA - Canthecona annulipes Dallas, 1851; syntype 9 ; labels: blue-margined syntype disc label, "Int. Africa / 4319", "Canthecona figurata Walker's catal.", "a”, "NHMUK 010747805"; Fig. 8M-N; NHMUK 010747805.

SOUTH AFRICA • Canthecona miniatescens Stål, 1854; syntype ; labels: "Caffraria", "I. Vahlb", "miniatescens Stål type.", "Typus", "NHRS-GULI 000027293"; Fig. 8D; examined by photo; NHRSGULI 000027293.

\section{Diagnosis}

Scutellum longer than wide, postfrenal lobe enlarged; humeral pronotal angles slightly emarginated; male abdomen with setose patches on segments V and VI, parameres triangular, without two distinct lobes.

\section{Redescription}

BoDy. Elongate oval, with variable colour patterns from yellowish and brownish with yellow or red stripes (Fig. 8D, H, L, N) to purple, green and blue metallic uniform colours (Fig. 8B-C, F, J, P).

HEAD. Subrectangular, wider than long, punctured; mandibular plates equal or slightly longer than clypeus, with margin varying from straight to sinuous; antenna bearing thin setae, denser on third, fourth and fifth antennomeres, proportion of lengths of antennomeres: IV $>$ V $>$ II $>$ III $>$ I; labium robust, reaching metasternum; proportion of lengths of labiomeres: II $>$ I $>$ IV $>$ III. 


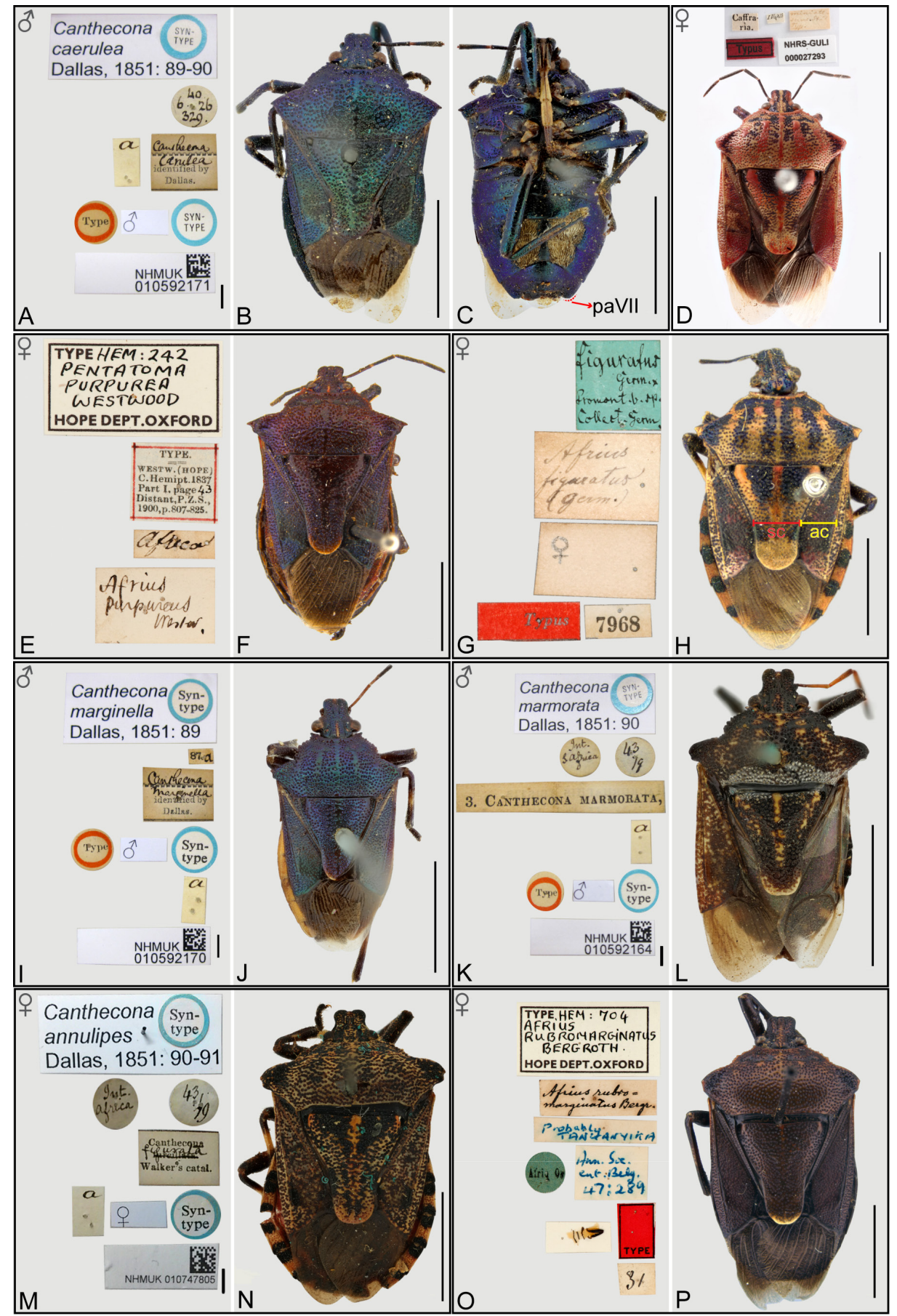

Fig. 8. Afrius (Afrius) purpureus (Westwood, 1837), type specimens. A-C. Canthecona caerulea Dallas, 1851, syntype $\widehat{\partial}$, labels, dorsal and ventral habitus, respectively (NHMUK). D. Canthecona miniatescens Stål, 1854, syntype + , labels and dorsal view (image courtesy of Gunvi Lindberg, NHRS). E-F. Pentatoma purpurea Westwood, 1837, syntype $q$, labels and dorsal habitus (OUMNH). G-H. Asopus figuratus Germar, 1838, syntype + , labels and dorsal habitus (MFNB). I-J. Canthecona marginella Dallas, 1851, syntype $\hat{O}^{\lambda}$, labels and dorsal habitus (NHMUK). K-L. Canthecona marmorata Dallas, 1851, syntype $\widehat{\partial}$, labels and dorsal habitus (NHMUK). M-N. Canthecona annulipes Dallas, 1851, syntype q, labels and dorsal habitus (NHMUK). O-P. Afrius rubromarginatus Bergroth, 1903, syntype $o$, labels and dorsal habitus (OUMNH). Abbreviations: $\mathrm{ac}=$ corium adjacent to the scutellum constriction; $\mathrm{paVII}=$ posterior angles of seventh abdominal segment; $\mathrm{sc}=$ scutellum constriction. Scale bars: $4 \mathrm{~mm}$. 
THORAx. Pronotum hexagonal, densely punctured, twice or more as wide as long, cicatrices flat; anterior margin concave; lateral margins strongly sinuous, crenulated on anterior half (Fig. 9), humeral angle slightly emarginated, anterior humeral portion varying from convex (Fig. 9A, C) to acute or spinose (Fig. 9B, D). Scutellum longer than wide, densely punctured. Corium longer than scutellum, usually not surpassing connexival segment $\mathrm{V}$, densely punctured.

Male abdomen. Setose patches present on male abdominal segments V and VI (Fig. 8C); parameres uniramous, triangular.

\section{Male}

MeAsuREMENTS $(\mathrm{n}=5)$. Head length $1.69 \pm 0.08$ (1.61-1.76); width $1.90 \pm 0.12(1.76-2.10)$; pronotum length $2.83 \pm 0.30$ (2.41-3.22); width $5.70 \pm 0.46$ (5.32-6.45); scutellum length $3.51 \pm 0.29$ (3.14$3.95)$; width $3.33 \pm 0.28$ (3.06-3.79); length of antennomeres: I $0.30 \pm 0.04(0.26-0.34)$, II $1.08 \pm 0.11$ (0.93-1.24), III $1.06 \pm 0.10$ (0.93-1.16), IV $1.26 \pm 0.11(1.12-1.39)$, V $1.22 \pm 0.09$ (1.12-1.31); length of labiomeres: I $0.93 \pm 0.12(0.79-1.12)$, II $1.06 \pm 0.09(0.94-1.09)$, III $0.80 \pm 0.09(0.67-0.94)$, IV 0.83 \pm 0.08 (0.71-0.94); length of abdomen $4.67 \pm 0.46$ (4.03-5.24); width $4.83 \pm 0.40$ (4.43-5.48); total length $10.03 \pm 0.52(9.35-10.64)$.

Genitalia. Genital plates cylindrical (Fig. 10A, C-D, F, I, gp). Parameres long, uniramous, head elongated triangular, dorsally directed, extended beyond pygophore (Fig. 10A-H, par). Phallus. Basal theca and thecal shield subequal in length (Fig. 10L-R, bt, ts). Vesica subtriangular in dorsal view (Fig. 10P-R, $\mathrm{v}$ ), golf club-shaped in lateral view, with apex obtuse and a central, strongly elevated portion covered by microsculptures (Fig. 10L-O, v, m), this central portion broad, rectangular in lateral view, cylindrical in dorsal view; ductus seminis uniform (Fig. 10L-R, ds), dorsally directed. Apices of conjunctival lobes globose, endowed with a set of small sculptured processes (Fig. 10L, N, O, cl, sp).

\section{Female}

Measurements $(\mathrm{n}=5)$. Head length $2.12 \pm 0.19(1.83-2.28)$; width $2.12 \pm 0.12$ (1.91-2.21); pronotum length $3.17 \pm 0.20$ (2.82-3.30); width $6.94 \pm 0.31$ (6.45-7.25); scutellum length $4.43 \pm 0.39$ (3.79-4.75); width $4.06 \pm 0.34$ (3.46-4.35); lengths of antennomeres: I $0.38 \pm 0.04(0.34-0.45)$, II $1.30 \pm 0.15$ (1.12-1.42), III $1.17 \pm 0.14$ (0.93-1.31), IV $1.50 \pm 0.19$ (1.16-1.61), V $1.37 \pm 0.15$ (1.12-1.50); lengths of labiomeres: I $1.04 \pm 0.07$ (0.94-1.12), II $1.27 \pm 0.15$ (1.01-1.38), III $0.93 \pm 0.09$ (0.78-1.01), IV 0.94 \pm 0.11 (0.75-1.05); length of abdomen $6.17 \pm 0.34$ (5.64-6.45); width $6.27 \pm 0.42$ (5.64-6.61); total length $11.96 \pm 0.83(10.80-13.06)$.

GenITALIA. Gonocoxites VIII slightly wider than long, posterior margins sinuous (Fig. 11A-B, gcVIII). Median and inner duct of vesicular area of uniform diameter (Fig. 11C-D, md, id).
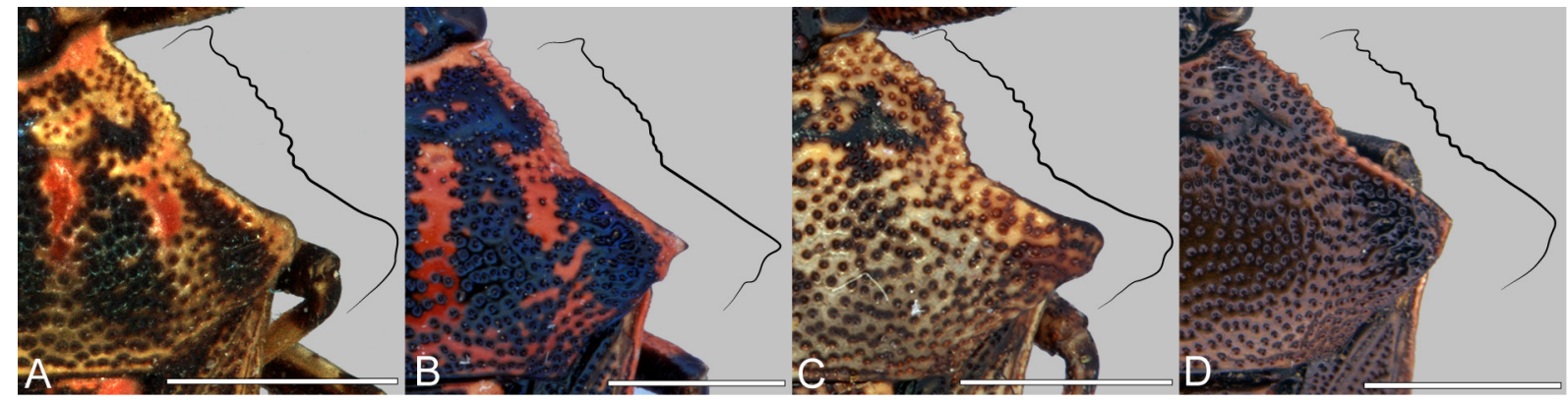

Fig. 9. Afrius (Afrius) purpureus (Westwood, 1837), variation of the pronotum shape. Scale bars: $2 \mathrm{~mm}$. 

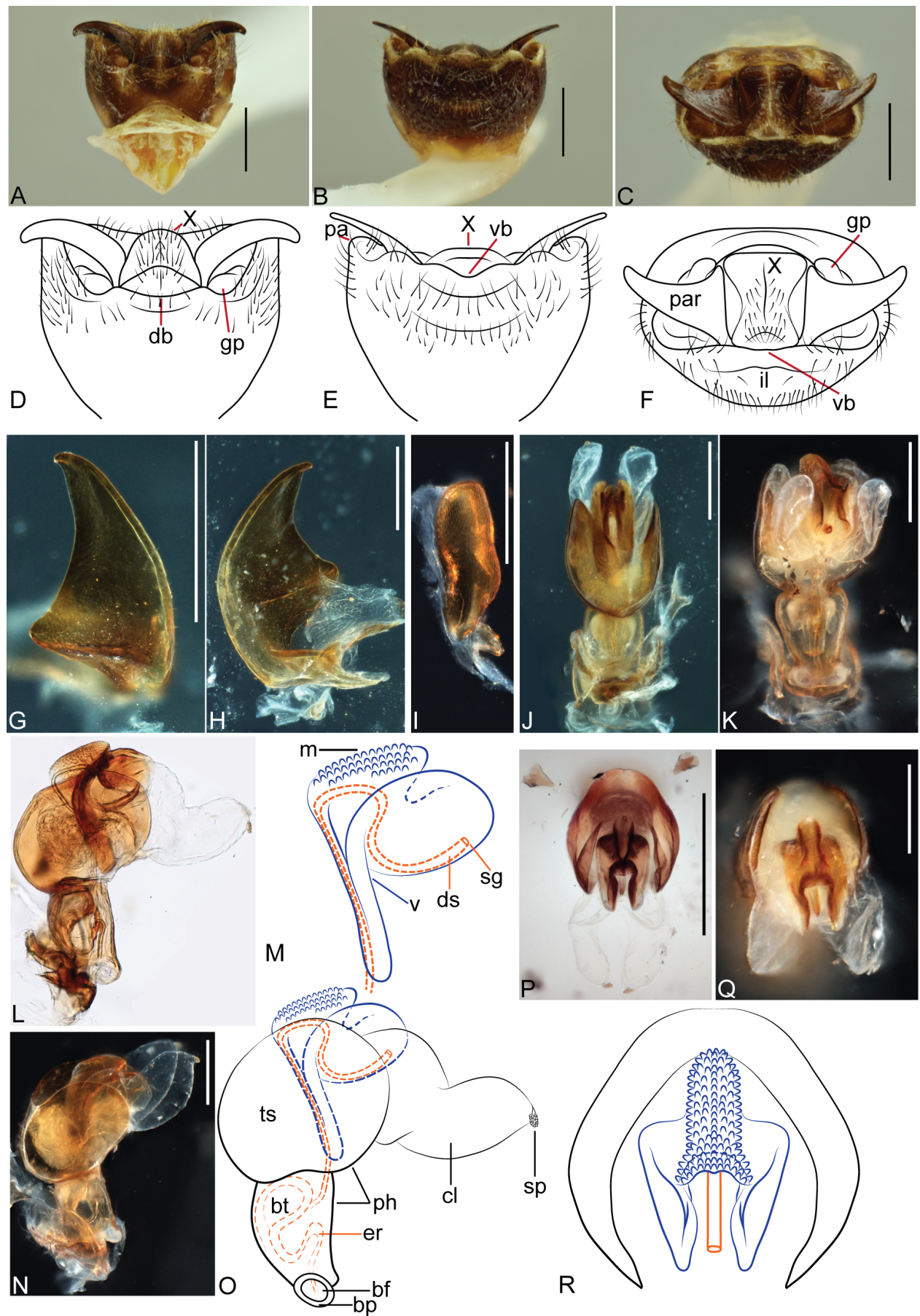

Fig. 10. Afrius (Afrius) purpureus (Westwood, 1837), male genitalia. A-F. Pygophore in dorsal (A, D), ventral $(\mathrm{B}, \mathrm{E})$ and posterior $(\mathrm{C}, \mathrm{F})$ views. G-H. Right paramere in lateral views, internal and external respectively. I. Right genital plate, dorso-posterior view. J-L. Phallus in anterior, posterior and lateral views, respectively. M. Detail of the vesica in lateral view, also represented in O. N. Phallus in lateral view. O. Drawing of the phallus. P-R. Phallus in dorsal view. Abbreviations: $b f=$ basal foramen; $b p=$ basal plates; $\mathrm{bt}=$ basal theca; $\mathrm{cl}=$ conjunctival lobes; $\mathrm{db}=$ dorsal border; $\mathrm{ds}=$ ductus seminis; $\mathrm{er}=$ ejaculatory reservoir; $\mathrm{gp}=$ genital plates; $\mathrm{il}=$ inferior layer; $\mathrm{m}=$ microsculptures; $\mathrm{pa}=$ posterolateral angles; $\mathrm{par}=$ parameres; $\mathrm{ph}=$ phallotheca; $\mathrm{sg}=$ secondary gonophore; $\mathrm{sp}=$ conjunctival process; $\mathrm{ts}=$ thecal shield; $v=$ vesica; $v b=$ ventral border; $X=$ segment $X$. Scale bars: $A-C=0.5 \mathrm{~mm}$; G-K, N, $\mathrm{P}-\mathrm{Q}=0.25 \mathrm{~mm}$. 


\section{Distribution}

South Africa (Germar 1838), Senegal (Guérin-Méneville 1844), Sierra Leone (Dallas 1851), Guinea (Stål 1864), Equatorial Guinea [as Fernando Po] (Stål 1870), Ghana (Reuter 1882), Mozambique (Gerstaecker 1892), Zimbabwe [as Mashonaland] (Distant 1898), Democratic Republic of the Congo (Distant 1901), Equatorial Guinea [as Fernando Po] (Stål 1870), Ghana (Reuter 1882), Mozambique (Gerstaecker 1892), Benin, Cameroon, Central African Republic [as Haute-Sangha], Eritrea, Ethiopia

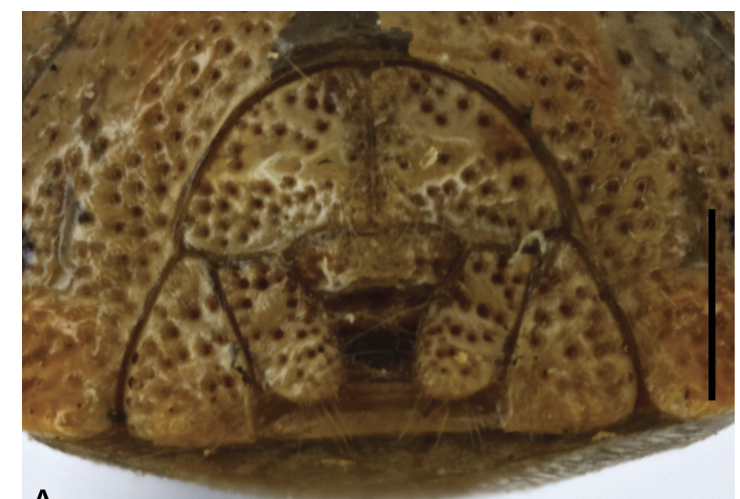

A

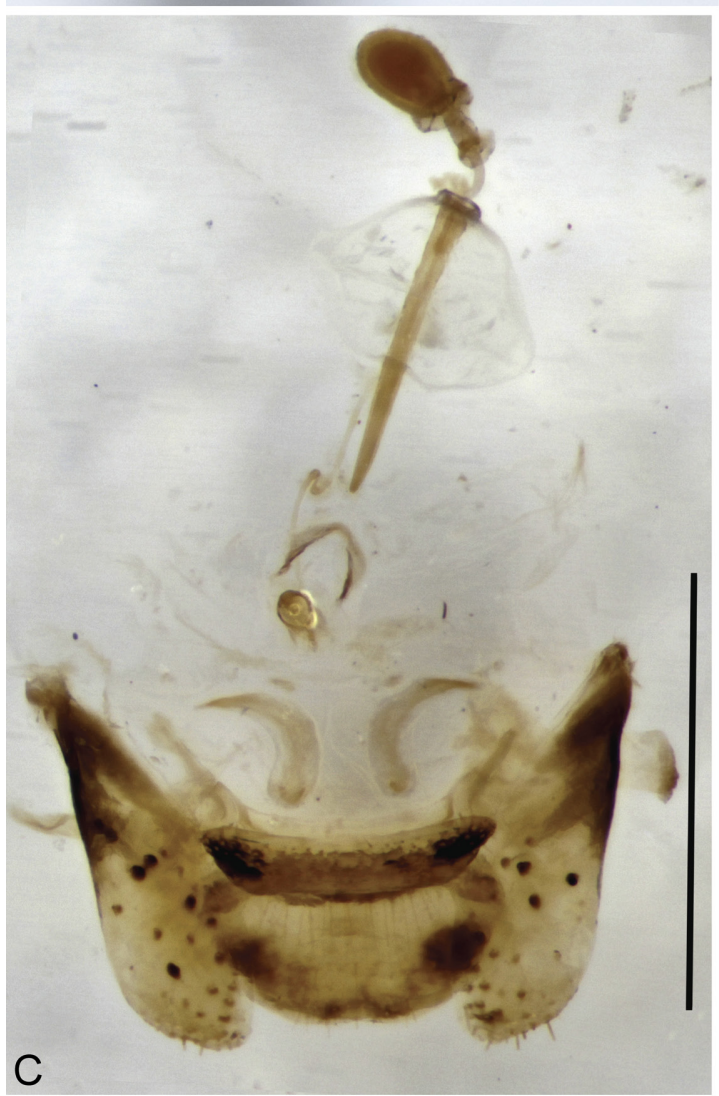

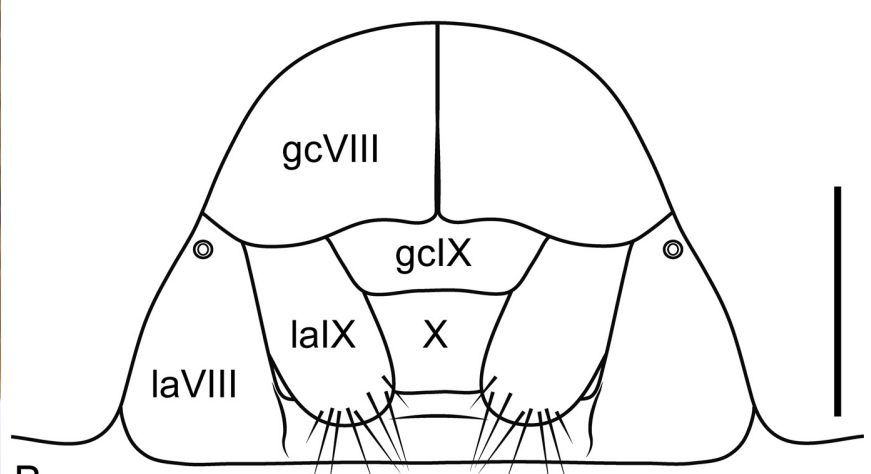

B

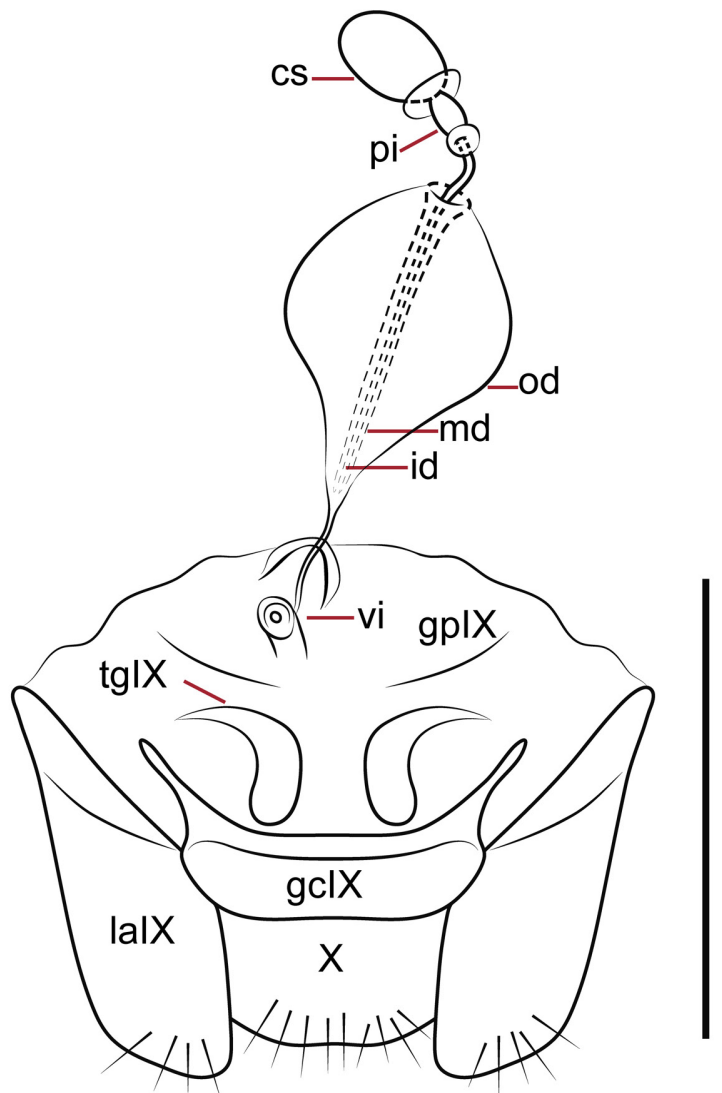

Fig. 11. Afrius (Afrius) purpureus (Westwood, 1837), female genitalia. A-B. Genital plates, ventroposterior view. C-D. Inner genitalia, ventral view. Abbreviations: $\mathrm{cs}=$ capsula seminalis; gcVIII = gonocoxites VIII; gcIX = gonocoxites IX; gpIX = gonapophyses IX; id = inner duct; laVIII = laterotergites VIII; laIX = laterotergites IX; $\mathrm{md}=$ median duct of vesicular area; od = outer duct of vesicular area; $\mathrm{pi}=$ pars intermedialis; $\operatorname{tgIX}=$ secondary thickening of gonapophyses IX; vi = thickening of vaginal intima; $\mathrm{X}=$ segment X. Scale bars: A-B $=1 \mathrm{~mm} ; \mathrm{C}-\mathrm{D}=0.5 \mathrm{~mm}$. 
[as Abyssinia], Gabon, Ivory Coast [as Grand Bassam], Nigeria [as Benue Niger], Sierra Leone, South Sudan, Sudan, Tanzania [as Usambara and Kilimanjaro] (Schouteden 1905a), Kenya [as Leitokitok] (Schouteden 1910), Mali [as Koulikoro] (Vuillet \& Vuillet 1911), Guinea-Bissau (Schouteden 1913b), Namibia [as Damaraland, Otjiwarongo and Tsumeb] (Hesse 1925), Uganda (Carpenter 1926), Somalia (Mancini 1937), Cape Verde Islands (Lindberg 1958 or 1959?), Chad (Couilloud 1989), Yemen (Linnavuori 1989), Angola, Botswana, Gambia, Liberia (Thomas 1994), Malawi (Sileshi et al. 2000), Zambia (Sileshi et al. 2001), Republic of Djibouti, Togo (Maldès \& Pluot-Sigwalt 2004), Republic of the Congo [as Haut Oubangui?] (Maldès \& Pluot-Sigwalt 2004; also in Robertson 2009 as "Congo Brazz.”), Niger (Robertson 2009; in error?), Canary Islands (new record) (Table 1, Fig. 1).

\section{Remarks}

Intraspecific variability in the colour and general morphology has been demonstrated for A. (Afrius) purpureus (e.g., Linnavuori 1989; Schouteden 1905a; Van Heerden 1931; Villiers 1952b) as well as for other pentatomids, such as Nezara viridula (Linnaeus, 1758) (e.g., Freeman 1940; Kiritani 1970; Ohno \& Alam 1992; Vivan \& Panizzi 2002; More et al. 2017) and Stiretrus decemguttatus (Lepeletier \& Serville, 1828) (Paleari 2013) for colour pattern, and Pinthaeus sanguinipes (Fabricius, 1781) for variation in the pronotum, reported by Zhao et al. (2013). Besides, we could not find any connection between the polymorphism and the geographic distribution of $A$. (Afrius) purpureus, i.e., specimens that we have examined, despite different colour patterns and pronotum shapes, are sympatric. The localities where they were collected are denoted by the green dots on Fig. 1.

There has been considerable difference of opinion regarding the name used for this species, i.e., either yolofal-us, purpureal-us or figuratal-us and, sometimes, the latter used as a variety or subspecies of the second one (see review of the taxonomic history of the species above). Dallas (1851) was the first to synonymize figuratus and yolofa; he stated the date of publication of Pentatoma yolofa as 1830, while Stål (1864) stated it as 1829. Subsequent authors (including Stål 1870) accepted 1838 as the year of publication for yolofa and therefore they recognized either figuratus or purpureus as the valid name, Wallengren (1875) may have been the last author to use yolofa (misspelled as Ylofa) as a valid name while Larousse (1890) gave a brief description of it under the genus Canthecona (strangely, this entry first mentions that the type species of Canthecona is from Senegal and thereafter describes C. yolofa, as though implying it were the type species; the type species of Canthecona Amyot \& Serville, 1843 is, however, C. discolor (Palisot de Beauvois, 1811), described from the Kingdom of Oware, now Southwest Nigeria). Dupuis (1952) demonstrated the priority of Pentatoma yolofa Guérin-Ménéville, 1831 over Pentatoma purpurea Westwood, 1837 and Asopus figuratus Germar, 1838. Thomas (1994) adopted the combination suggested by Dupuis (1952), Afrius yolofus. This was, as well, used by subsequent authors (Maldès \& Pluot-Sigwalt 2004; Rebagliati et al. 2005; Kerisew 2011). Some others (Sileshi et al. 2001, 2004; Kerzhner et al. 2004; Rider 2006; Robertson 2009; Matesco et al. 2014), however, chose the combination Afrius yolofa, while others again still used purpureus and/or figuratus after 1952 (e.g., Mancini 1953; Villiers 1954, 1956; Leston 1954; Lindberg 1958; Schouteden 1963, 1964; Gillon 1972; Linnavuori 1975, 1976, 1982; Bourdouxhe \& Jolivet 1981; Nuamah 1982; Matanmi \& Hassan 1987; Balsbaugh 1988; Couilloud 1989; Quicke et al. 1992; Schaefer 1996; Tchibozo \& Braet 2004; Poutouli et al. 2011; Agboton et al. 2014; Crétenet \& Gourlot 2015) and yet others treated figuratus as a variety or a subspecies of purpureus (Schouteden 1905a, 1907a; Kirkaldy 1909; Schouteden 1909; Mancini 1937; Le Pelley 1959; Linnavuori 1989). Others still have used two or three combinations in the same work, possibly because they were reporting facts from primary sources, using the names as they were in the sources, and were not aware that the species were the same (Le Pelley 1959; Schaefer 1996).

The different combinations used for one and the same species clearly are the results of a few misconceptions: when and how yolofa, figuratus and purpureus were synonymized, the problematic dating of yolofa and figuratus and the status of yolofa as an adjective or a noun in apposition. 


\section{Synonymies of yolofa, figuratus and purpureus}

Earlier we noted that Dallas (1851) first stated the synonymy of figuratus with yolofa. Clearly, Stål (1870) and Schouteden (1905a) believed yolofa sensu Dallas (1851) was a misidentification, pertaining to figuratus. Pentatoma yolofa was synonymized with Cimex (Afrius) purpureus by Stål (1870). Asopus figuratus was considered as a variety of Canthecona purpurea by Schouteden (1905a). It appeared as a junior synonym of Afrius yolofus together with Pentatoma purpurea in Dupuis (1952). Later, Leston (1954) made use of it as the valid name of the species; yet, in a footnote, it was corrected that the valid name should be Afrius yolofus. Additionally, the confusion between the use of figuratus and purpureus has been so great that some authors have even attributed the authorship of purpureus to Germar (e.g., Risbec 1950; Herting 1971, 1976). Considering the above, it is no surprise that figuratus appeared as a variety or subspecies of purpureus, even relatively recently. It is no surprise either that it still appeared as a valid name as late as 2000 (Sileshi et al. 2000) and possibly later, concurrently with the other names (yolofa, yolofus and purpureus).

\section{Problematic dating of yolofa and figuratus}

As stated above, until Dupuis (1952), yolofa had been considered as a junior synonym of purpureus as its date of publication was thought to be 1838 . This actually is that of figuratus. For a long time there was considerable confusion about the priority of the works of Westwood (1837) and Germar (1838); the title pages of both works indicated 1837 as the date of publication. Schouteden (1907b) demonstrated that Westwood's work had priority. Sherborn (1922-1932) attributed the date "1840" to all taxa published by Germar in the fifth volume of the Revue entomologique (pages 121-192); most modern catalogues of Heteroptera list Germar's work with the date 1838 (e.g., Rolston et al. 1993; Schuh 1995; Aukema \& Rieger (eds) 1995-2013; Rolston et al. 1996; Cassis \& Gross 2002; CoreoideaSF Team 2018; Dellapé \& Henry 2018). Recently, Nagel \& Schmidlin (2014: 97) stated a precise date (21 November 1838) for taxa newly described between pages 1 to 224 of the fifth volume. As a consequence, Pentatoma purpurea Westwood, 1837 definitely has priority over Asopus figuratus Germar, 1838 [not 1837 as earlier authors had assumed], and Pentatoma yolofa Guérin-Méneville, 1831 over them both.

\section{Should we use yolofa, a noun in apposition or yolofus, an adjective?}

The original description indubitably shows that Guérin-Méneville chose an adjective and not a noun in apposition as may have been thought by recent authors who made use of the combination Afrius yolofa, possibly influenced by earlier authors (Amyot \& Serville 1843; Dallas 1851).

Guérin-Méneville (1831: plate 55, 1844: 344) used the binomen Pentatoma yolofa with a lower case " $y$ ", implying that the name was treated as an adjective (a capital "D" was used for Scutellera Dives and a capital "S" for Tesseratoma [sic] Sonneratii on the same plate, as one would expect in those days for a noun in apposition or a genitive based on the name of a person); the same author (Guérin-Méneville 1844: 344) also used the French vernacular name P[entatome] yolofe. Amyot \& Serville (1843) cited this species as Canthecona Yolofa (with vernacular French as Canthécone Yolofa), using a capital "Y" in the Latin binomen (also followed by Dallas 1851), and a final "a" in the French name. Their use of a capital "Y" and of a final "a" in the French name indicates that these subsequent authors treated the species name as a noun in apposition.

The Grand Dictionnaire Universel du XIX siècle (Larousse 1876: 1423) has an entry for the adjective "YOLOF ou YOLOFF, OVE", meaning "relative to the native language of the Wolof people (Senegal, Gambia, Mauritania)". Although the adjective is there restricted to the field of linguistics and despite the fact that the proper feminine form of the adjective in French is "yolove", it is clear that GuérinMéneville (1831) meant to indicate that his Pentatoma, collected in Senegal, the land of the wolof/yolof people, was 'yolove' (expressed with an adjective), so he called it Pentatome yolofe/Pentatoma yolofa. Admittedly, this adjective has not been used much in zoology; Sherborn (1932: 7031) only lists one 
other species having yolofus as its epithet, Prionus yolofus (Dalman, 1817) (Coleoptera: Cerambycidae: Prioninae: Acanthophorini). The latter species is now placed in the genus Tithoes Thomson, 1864, which contains two other species whose epithets equally express their rather precise African provenance: Tithoes congolanus (Lameere, 1903) and T. somalius (Lameere, 1903).

The precedence of $A$. yolofa over its synonyms, pointed out by Dupuis (1952) and echoed by Leston (1954), was generally ignored until Thomas (1994). Even now, few are those who apply it. In almost two centuries, a substantial number of papers on the species have been published using either purpureus or figuratus. Since purpureus has been the most used overall, since it was used continually to refer to this species since its publication and since Afrius purpureus is the name used in the latest publication on the species that we are aware of, we have used the name Afrius purpureus in this revision as the valid name. In addition, we intend to apply to the International Commission on Zoological Nomenclature, under Article 23.9.3 of the Code (ICZN 1999), so that the prevailing usage of the specific name Pentatoma purpurea Westwood, 1837 (currently Afrius purpureus) be conserved and ensured in future.

\section{Discussion}

The idea that insects can control agricultural pests is old, and the success of biological control has already been demonstrated in many countries (Caltagirone 1981; McFadyen 1998; Bellows 2001; Parra et al. 2002). The predatory stink bugs that make up the subfamily Asopinae are among the insects with potential use in biological control (Magistrali et al. 2014), but despite this, there are still few modern phylogenetic and taxonomic studies on this subfamily. The lack of taxonomic studies may result in great economic losses when controlling and controlled species are poorly identified (Zucchi 2002). In this work we revised an important genus of Asopinae for biological control, namely Afrius Stål, 1870 (Miller 1952; Sileshi et al. 2004), which is distributed throughout Africa and adjacent islands and comprises, after this revision, three species: Afrius (Subafrius) flavirostrum (Signoret, 1861), Afrius (Afrius) kolleri Schouteden, 1911 and Afrius (Afrius) purpureus (Westwood, 1837). The contributions presented here could serve in future phylogenetic and taxonomic studies, as well as in applied agronomical sciences.

We maintained the two subgenera which were proposed by Schouteden (1907a) as they show distinct characteristics, such as the absence of abdominal glandular patches on males of Afrius (Subafrius) flavirostrum and the parameres which are divided into two rami in the same species. Perhaps a phylogenetic scenario including other similar genera can better substantiate this classification in the future.

We demonstrated in this study the importance of consulting type materials in taxonomy. For example, Miller (1952) had probably not seen the type material of $A$. (Subafrius) flavirostrum when he described the new species $A$. williamsi Miller, 1952, which we are synonymizing here. Furthermore, we strongly recommend the study of the external and internal morphology of the genitalia in Asopinae, as these were crucial here to the delimitation of species, especially of Afrius (Afrius) purpureus, which shows a broad geographical distribution and different patterns of general morphology and colour (Linnavuori 1989; Schouteden 1905a; Van Heerden 1931; Villiers 1952b).

We cannot hypothesize the relationship of the species of Afrius without a phylogenetic study, and at present we do not know if the genus is monophyletic. Afrius (Afrius) kolleri and A. (A.) purpureus seem to be more closely related to each other than to A. (Subafrius) flavirostrum based on similar male genitalia, i.e., the presence of vesica microsculptures and the presence of male abdominal glandular patches.

Afrius (Afrius) purpureus is a species with a broad geographical distribution and a great intraspecific variability with respect to size, morphology of the head and pronotum, and colour pattern (Figs 8-9). 
Because of the observed differences we consider the morphology of the genitalia crucial to delimit the species of Afrius.

\section{Acknowledgements}

Herbert Zettel, Harald Bruckner (NHMW, Vienna) and Gunvi Lindberg (NHRS, Stockholm) sent images of types and their labels as well as other information about them. Dominique Pluot-Sigwalt (MNHN, Paris) and Angela Thresher (NHMUK, London) helped us to obtain important references. Jürgen Deckert (MFNB, Berlin), Jérôme Constant (Royal Belgian Institute of Natural Sciences, Brussels), Stéphane Hanot (RMCA, Tervuren), Eric Guilbert (MNHN, Paris) and Amoret Spooner (OUMNH, Oxford) received the first author in their collections and provided material for this study. Randall T. Schuh and Ruth Salas (AMNH, New York), David A. Rider (North Dakota State University, Fargo), Kurt Arnold (Entomologisches Museum - Insekten Dauerausstellung, Geyer), and Petr Kment (NMPC, Prague) also provided material for this study. Luiz Alexandre Campos (UFRG, Porto Alegre) made suggestions regarding the terminology of genitalia structures. We thank them all. We also thank Anna Namyatova (Saint Petersburg University, Saint Petersburg), Jocélia Grazia (UFRG, Porto Alegre), and Dávid Rédei (Nankai University, Tianjin), whose helpful comments improved our manuscript. We are particularly grateful to CAPES (Brazil) for having funded the research of TR under fellowship grant PDSE - 88881.133880/2016-01.

\section{References}

Agboton C., Onzo A., Ouessou F.I., Goergen G., Vidal S. \& Tamò M. 2014. Insect fauna associated with Anacardium occidentale (Sapindales: Anacardiaceae) in Benin, West Africa. Journal of Insect Science 14 (1): 1-11. https://doi.org/10.1093/jisesa/ieu091

Amyot C.J.B. \& Serville A. 1843. Histoire naturelle des insectes. Hémiptères. Roret, Paris. https://doi.org/10.5962/bhl.title.8471

Arechavaleta M., Zurita N., Marrero M.C. \& Martín J.L. (eds) 2005. Lista preliminar de Especies silvestres de Cabo Verde (Hongos, Plantas y Animales terrestres). Consejería de Medio Ambiente y Ordenación Territorial, Gobierno de Canarias.

Aukema B. \& Rieger C. (eds). 1995-2013. Catalogue of the Heteroptera of the Palaearctic Region [in six volumes]. Netherlands Entomological Society, Amsterdam.

Baker A.D. 1931. A study of the male genitalia of Canadian species of Pentatomidae. Canadian Journal of Research 4: 181-220. https://doi.org/10.1139/cjr31-013

Balsbaugh E.U. 1988. 16. Mimicry and the Chrysomelidae. In: Jolivet P., Petitpierre E. \& Hsiao T.H. (eds) Biology of Chrysomelidae: 261-284. Series Entomologica 42, Kluwer Academic Publisher, Dordrecht/Boston/London.

Beenen R. \& Hawkeswood T.J. 2004. Cydippa balyi Chapuis, 1875 (Coleoptera, Chrysomelidae), an interesting galerucine from Australia rediscovered in the Northern Territory, with redescription and notes on its habitat and host plant. In: Jolivet P., Santiago-Blay J.A. \& Schmitt M. (eds) New Developments in the Biology of Chrysomelidae: 469-473. SPB Academic Publishing, The Hague.

Bellows T.S. 2001. Restoring population balance through natural enemy introductions. Biological Control 21: 199-205. https://doi.org/10.1006/bcon.2001.0936

Bergroth E. 1903. Rhynchota Aethiopica III. Annales de la Société entomologique de Belgique 47: 288-297.

Bergroth E.E. 1908. Enumeratio Pentatomidarum post Catalogum bruxellensem descriptarum. Mémoires de la Société entomologique de Belgique 15 (10): 131-200. 
Bijlmakers H.W.L. \& Verhoek B.A. 1995. Guide de Défense des Cultures au Tchad. Cultures vivrières et maraîchères. Food and Agriculture Organization of the United Nations, Rome.

Boulard M. 1998. Abécédaire illustré du Mimétisme. École pratique des Hautes Études: Biologie et Évolution des Insectes 10 [1997]: 3-77.

Bourdouxhe L. \& Jolivet P. 1981. Nouvelles observations sur le complexe mimétique de Mesoplatys cincta Olivier (Coléoptère Chrysomelidae) au Sénégal. Bulletin Mensuel de la Société Linnéenne de Lyon 50 (2): 46-48. https://doi.org/10.3406/linly.1981.10464

Brinck P. 1955. Swedish exploration of South African animal life during 200 years. In: Hanstrom B., Brinck P. \& Rudebeck G. (eds) South African Animal Life. Results of the Lund University Expedition in 1950 - 1951. Vol. I: 11-61. Almqvist \& Wiksell, Stockholm. [Not seen, fide Ruta \& Libonatti 2016: 206]

Cachan P. 1952. Les Pentatomidae de Madagascar (Hémipteres Hétéropteres). Mémoires de l'Institut scientifique de Madagascar (E) 1 (2): 231-462.

Cachan P. 1965. Morphologie et biologie de Afrius purpureus Westwood, 1837 Asopinae prédateur dans la région de Dakar (Hétéroptères-Pentatomidae). Annales de la Faculté des Sciences de l'Université de Dakar 18: 5-32.

Caltagirone L.E. 1981. Landmark examples in classical biological control. Annual Review of Entomology 26: $213-232$.

Carpenter D.H. 1926. Various observations on insects in Uganda. Transactions of the Entomological Society of London 58: liii-lviii.

Cassis G. \& Gross G.F. 2002. Hemiptera: Heteroptera (Pentatomomorpha). In: Houston W.W.K. \& Wells A. (eds) Zoological Catalogue of Australia. Vol. 27.3B. CSIRO Publishing, Melbourne, Australia.

Caswell G.H. 1962. Agricultural Entomology in the Tropics. Edward Arnold Ltd., London.

Cobben R.H. 1968. Evolutionary Trends in Heteroptera. Part I. Eggs, Architecture of the Shell, Gross Embryology and Eclosion. PhD thesis, Agricultural College, Wageningen, the Netherlands. Available from https://edepot.wur.n1/187075 [accessed 13 May 2018].

CoreoideaSF Team. 2018. Coreoidea Species File Online. Version 5.0/5.0.

Available from http://Coreoidea.SpeciesFile.org [accessed 30 May 2018].

Couilloud R. 1989. Hétéroptères déprédateurs du cotonnier en Afrique et à Madagascar (Pyrrhocoridae, Pentatomidae, Coreidae, Alydidae, Rhopalidae, Lygaeidae). Coton et Fibres tropicales 44 (3): 185-226.

Cox M.L. 1996. Insect predators of Chrysomelidae. In: Jolivet P.H.A. \& Cox M.L. (eds) Chrysomelidae Biology. Vol. 2: Ecological Studies: 23-91. SPB Academic Publishing, Amsterdam.

Crétenet M. \& Gourlot J.P. (eds) 2015. Le Cotonnier. Quae, CTA, Presses agronomiques de Gembloux, Gembloux.

Dallas W.S. 1851. List of Specimens of Hemipterous Insects in the Collection of the British Museum, Pt. 1. Taylor \& Francis, London. https://doi.org/10.5962/bhl.title.20373

Dellapé P.M. \& Henry T.J. 2018. Lygaeoidea Species File. Version 5.0/5.0.

Available from http://Lygaeoidea.SpeciesFile.org [accessed 30 May 2018].

Distant W.L. 1884. On the Rhynchota collected by the late Mr. W.A. Forbes on the Lower Niger. Proceedings of the Zoological Society of London 1884 (3): 458-461.

Distant W.L. 1890. Ethiopian Rhynchota in the collection of the Brussels Museum. Annales de la Société entomologique de Belgique 34: li-1xi. 
Distant W.L. 1892. A Naturalist in the Transvaal. R.H. Porter, London. https://doi.org/10.5962/bhl.title.2478

Distant W.L. 1898. Rhynchota from the Transvaal, Mashonaland, and British Nyasaland. Annals and Magazine of Natural History (7) 2: 294-316. https://doi.org/10.1080/00222939808678047

Distant W.L. 1901. On the Rhynchota of the Congo region (Part I). Annales de la Société entomologique de Belgique 45: 23-31.

Distant W.L. 1913. Percy Sladen Trust Expedition to the Seychelle Islands. No. IX. Rhynchota. Part I: Suborder Heteroptera. Transactions of the Linnean Society of London 16 (2): 139-191. https://doi.org/10.1111/j.1096-3642.1913.tb00115.x

Dolling W.R., Rider D.A. \& Rolston L.H. 1999. Catalog of the Names of Cimex Linnaeus, With Comments on Early Works Concerning the Heteroptera. Thomas Say Publications in Entomology, Monographs, Entomological Society of America, Lanham.

Dupuis C. 1952. Priorité de quelques noms d'Hétéroptères de Guérin Méneville (1831). Bulletin de la Société zoologique de France. 77: 447-454.

Dupuis C. 1955. Les génitalia des Hémiptères Hétéroptères (Génitalia externes des deux sexes; Voies ectodermiques femelles). Mémoires du Muséum national d'Histoire naturelle, Série A, Zoologie 6 (4): $183-278$.

Dupuis C. 1970. Heteroptera. In: Tuxen S.L. (ed.) Taxonomist's Glossary of Genitalia of Insects: 190208. Munksgaard, Copenhagen.

Freeman P. 1940. A contribution to the study of the genus Nezara Amyot and Serville Hemiptera, Pentatomidae. Transactions of the Royal Entomological Society of London 90: 351-374.

Fry J.M. 1989. Natural Enemy Databank 1987: A Catalogue of Natural Enemies of Arthropods derived from Records on the CIBC Natural Enemy Databank. CAB International [Institute of Biological Control], Wallingford.

Gapon D.A. \& Konstantinov F.V. 2006. On the structure of the aedeagus of shield bugs (Heteroptera: Pentatomidae): III. Subfamily Asopinae. Entomological Review 86 (7): 806-819.

Germar E.F. 1838. Hemiptera Heteroptera Promontorii Bonae Spei, nundum descripta, quae collegit C.F. Drège, et proponit E.F. Germar. Revue entomologique (Silbermann) 5: 121-192.

Gerstaecker A. 1892. Bestimmung der von Herrn. Dr. F. Stuhlmann in Ostafrika gesammelten Hemiptera. Jahrbuch der Hamburgischen Wissenschaftlichen Anstalten 9 (2): 45-58.

Gillon D. 1972. Les Hémiptères Pentatomides d'un savane préforestière de Côte-d'Ivoire. Annales de l'Université d'Abidjan (E) 5 (1): 265-371.

Gillon D. 1974. Étude biologique des espèces d'Hémiptères Pentatomides d'une savane préforestière de Côte d'Ivoire. Annales de l'Université d'Abidjan (E) 7 (1): 213-303.

Girard D.H. 1969. List of Intercepted Plant Pests, 1968. (Pests Recorded From July 1, 1967, Through June 30, 1968). United States Department of Agriculture - Agricultural Research Service - Plant Quarantine Division, Hyattsville, Maryland, USA.

Golding F.D. 1931. Further notes on the food-plants of Nigerian insects. Bulletin of Entomological Research 22 (2): 221-223. https://doi.org/10.1017/S0007485300035185

Grazia J., Panizzi A.R., Greve C., Schwertner C.F., Campos L.A., Garbelotto T.A. \& Fernandes J.A.M. 2015. Stink bugs (Pentatomidae). In: Panizzi A. \& Grazia J. (eds) True Bugs (Heteroptera) of the Neotropics: 681-756. Entomology in Focus 2. Springer, Dordrecht.

https://doi.org/10.1007/978-94-017-9861-7_22 
Guérin-Méneville F.E. 1831 [1829-1838]. Iconographie du Règne Animal de G. Cuvier, ou Représentation d'après Nature de l'une des Espèces les plus remarquables et souvent non encore figurées, de chaque Genre d'Animaux. Vol. 1: Insectes. J.B. Baillère, Paris and London.

Guérin-Méneville F.E. 1844 [1829-1844]. Iconographie du Règne Animal de G. Cuvier, ou Représentation d'après Nature de l'une des Espèces les plus remarquables et souvent non encore figurées, de chaque Genre d'Animaux. Vol. 2: Planche des Animaux invertébrés. J. B. Baillère, Paris and London.

Hargreaves E. 1937. Some insects and their food-plants in Sierra Leone. Bulletin of Entomological Research 28 (3): 505-520. https://doi.org/10.1017/S0007485300038979

Herrich-Schaeffer G.A.W. 1844. Die Wanzenartigen Insecten. Vol. 7. C.H. Zeh'schen Buchhandlung, Nuremberg.

Herting B. 1971. A Catalogue of Parasites and Predators of Terrestrial Arthropods. Section A - Host or Prey/Enemy. Vol. I - Arachnida to Heteroptera. Commonwealth Agricultural Bureaux, Slough.

Herting B. 1973. A Catalogue of Parasites and Predators of Terrestrial Arthropods. Section A - Host or Prey/Enemy. Vol. III - Coleoptera to Strepsiptera. Commonwealth Agricultural Bureaux, Slough.

Herting B. 1976. A Catalogue of Parasites and Predators of Terrestrial Arthropods. Section A - Host or Prey/Enemy. Vol. VII - Lepidoptera, Part 2 (Macrolepidoptera). Commonwealth Agricultural Bureaux, Slough.

Hesse A. J. 1925. 1. Contributions to a knowledge of the fauna of South-West Africa. IV. A list of the Heteropterous and Homopterous Hemiptera of South-West Africa. Annals of the South African Museum 23: $1-190$.

Hilgert C. 1908. Katalog der Collection von Erlanger in Nieder-Ingelheim a. Rh. R. Friedlaender \& Sohn, Berlin. https://doi.org/10.5962/bhl.title.7511

Hollrung M. 1912. Jahresbericht über das Gebiet der Pflanzenkrankheiten. P. Parey, Berlin.

Howard C.W. 1906. The orange-tree butterfly: Papilio demoleus. Transvaal Agricultural Journal 4 (16): $727-732$.

ICZN. 1999. International Code of Zoological Nomenclature. Fourth Edition. The International Trust for Zoological Nomenclature, London.

Jeannel R. 1913. Pentatomidae In: Voyage de Ch. Alluaud et R. Jeannel en Africa Orientale (19111912). Résultats scientifiques. Insectes Hémipteres. Librairie Albert Schulz, Paris.

Jolivet P. 1997. Biologie des Coléoptères Chrysomélides. Société nouvelle des Éditions Boubée, Paris.

Jolivet P. \& Théodoridès P. 1953. Les parasites phorétiques et prédateurs des Chrysomeloidea (Coleoptera) (4 ${ }^{\mathrm{ième}}$ note). Bulletin de l'Institut royal des Sciences naturelles 29 (32): 1-15.

Kerisew B. 2011. Cytogenetic Investigations on Some Species of the Family Pentatomidae (Insecta: Hemiptera: Heteroptera). $\mathrm{PhD}$ thesis, Punjabi University, Patiala.

Available from http://hdl.handle.net/10603/3653 [accessed 9 May 2018].

Kerzhner I.M., Kuznetsova V.G. \& Rider D.A. 2004. Karyotypes of Pentatomoidea additional to those published by Ueshima, 1979 (Heteroptera). Zoosystematica Rossica 13 (1): 17-21.

Kiritani K. 1970. Studies on the adult polymorphism in the southern green stink bug Nezara viridula (Hemiptera: Pentatomidae). Researches on Populational Ecology 12: 19-34.

Kirkaldy G.W. 1909. Catalogue of the Hemiptera (Heteroptera) with Biological and Anatomical References, Lists of Foodplants and Parasites, etc. Vol. 1: Cimicidae. Felix L. Dames, Berlin. 
Kment P. \& Vilímová J. 2010. Thoracic scent efferent system of Pentatomoidea (Hemiptera: Heteroptera): a review of terminology. Zootaxa 2706: 1-77.

Konstantinov F.V. \& Gapon D.A. 2005. On the structure of the aedeagus of shield bugs (Heteroptera: Pentatomidae): 1. Subfamilies Discocephalinae and Phyllocephalinae. Entomological Review 85 (3): $221-235$.

Krüger A. \& Deckert J. 2016. True bugs (Hemiptera-Heteroptera) of Botswana-Bibliographical inventory and new records. Zootaxa. 4114 (1): 33-63. https://doi.org/10.11646/zootaxa.4114.1.2

Kuklinski F. \& Borgemeister C. 2002. Cotton pests and their natural enemies in Madagascar. Journal of Applied Entomology 126: 55-65. https://doi.org/10.1046/j.1439-0418.2002.00622.x

Laporte F.L. de. 1833. Essai d'une classification systématique de l'ordre des Hémiptères (HémiptèresHétéroptères Latr.). Magasin de Zoologie 2: 17-75, suppl. 76-88.

Larousse P. 1876. Grand Dictionnaire universel du XIXe Siècle: français, historique, géographique, mythologique, bibliographique, littéraire, artistique, scientifique, etc., Vol. 15: TESTAM-Z. Administration du grand Dictionnaire universel, Paris.

Larousse P. 1890. Grand Dictionnaire universel du XIXe Siècle: français, historique, géographique, mythologique, bibliographique, littéraire, artistique, scientifique, etc., etc... Vol. 17, Supplement 2. Administration du grand Dictionnaire universel, Paris.

Lehmann G. 1922. Erster Beitrag zur Verbreitung afrikanischer Pentatomiden (Heteroptera). (VII. Heteropteren-Aufsatz.). Konowia 1: 129-133.

Le Pelley R.H. 1959. Agricultural Insects of East Africa. East Africa High Commission, Nairobi.

Le Pelley R.H. 1968. Pests of Coffee. Longmans, Green and Co. Ltd, London and Harlow.

Leston D. 1952. Notes on The Ethiopian Pentatomoidea (Hemiptera). - VI. Some insects in the Hope Department, Oxford. Annals and Magazine of Natural History 12 (5): 893-904. https://doi.org/10.1080/00222935208654367

Leston D. 1954. Notes on the Ethiopian Pentatomoidea (Hem.) XII. On some specimens from Southern Rhodesia, with an investigation of certain features in the morphology of Afrius figuratus (Germar) and remarks upon the male genitalia in Amyotinae. Occasional Papers of the National Museum of Southern Rhodesia 19: 678-686.

Lethierry L. \& Severin G. 1893. Catalogue général des Hémiptères, Pentatomidae. Musée royal d'Histoire naturelle Belgique, Bruxelles.

Lindberg H. 1958. Hemiptera Insularum Caboverdensium. Systematik, Ökologie und Verbreitung der Heteropteren und Cicadiden der Kapverdischen Inseln. Commentationes Biologicae 19 (1): 1-246.

Linnavuori R. 1975. Hemiptera Heteroptera of the Sudan with remarks on some species of the adjacent countries. Part 5. Pentatomidae. Boletim da Sociedade Portuguesa de Ciências Naturais (2) 15: 5-128.

Linnavuori R. 1976. A hemipterous material from Somalia. Bollettino della Società Entomologica Italiana 108 (5-7): 123-130.

Linnavuori R.E. 1982. Pentatomidae and Acanthosomatidae (Heteroptera) of Nigeria and the Ivory Coast, with remarks on species of the adjacent countries in West and Central Africa. Acta Zoologica Fennica 163: 1-176.

Linnavuori R.E. 1989. Heteroptera of Yemen and south Yemen. Acta Entomologica Fennica 54: 1-40.

Magistrali I.C., Costa E.C., Machado L.M. \& Nadai J. 2014. Novos registros de Asopinae (Pentatomidae) predadores de lagartas Nystalea nyseus (Cramer, 1775) (Lepidoptera: Notodontidae). Biotemas 27 (2): 209-212. https://doi.org/10.5007/2175-7925.2014v27n2p209 
Maitai W.M. 1958. Annotated list of insects associated with the sweet potato (Ipomoea batatas). East African Agricultural Journal 23 (4): 290-291.

Maldès J.M. \& Pluot-Sigwalt D. 2004. Les Asopinae afrotropicaux et malgaches conservés au MNHN à Paris: Liste des spécimens types et des espèces représentées [Heteroptera: Pentatomidae]. Revue française d'Entomologie 26 (1): 19-28.

Mamet J. R. 1957. A revised and annotated list of the Hemiptera (Heteroptera and Homoptera, excluding Sternorrhyncha) of Mauritius. The Mauritius Institute Bulletin 5 (2): 31-81.

Mancini C. 1937. Resultati scientifici delle missioni del Prof. G. Paoli in Somalia. Emitteri - Nota I. Bollettino della Società Entomologica Italiana 69 (1-2): 40-44.

Mancini C. 1953. Missione biologica Sagan-Omo diretta dal Prof. Edoardo Zavattari (1939). HemipteraHeteroptera. Annali del Museo Civico di Storia Naturale Giacomo Doria 66:166-204.

Matanmi B.A. \& Hassan T.J. 1987. The life history and habits of Acraea eponina (Cramer) with notes on Acraea acerata Hewiston (Lepidoptera: Nymphalidae). Revue de Zoologie africaine 101 (3): 371-377.

Matesco V.C., Bianchi F.M., Fürstenau B.B.R.J., Da Silva P.P., Campos L.A. \& Grazia J. 2014. External egg structure of the Pentatomidae (Hemiptera: Heteroptera) and the search for characters with phylogenetic importance. Zootaxa 3768 (3): 351-385. https://doi.org/10.11646/zootaxa.3768.3.5

McDonald F.J.D. 1966. The genitalia of North American Pentatomoidea (Hemiptera: Heteroptera). Quaestiones Entomologicae 2: 7-150.

McFadyen R.E.C. 1998. Biological control of weeds. Annual Review of Entomology 43: 369-393.

Medler J.T. 1980. Insects of Nigeria: Check List and Bibliography. Memoirs of the American Entomological Institute 30, American Entomological Institute, Ann Arbor.

Merrett P.J. 1986. Natural enemies of the African armyworm, Spodoptera exempta (Walker) (Lepidoptera: Noctuidae), in Tanzania. Bulletin of Entomological Research 76 (4): 545-552.

https://doi.org/10.1017/S0007485300015054

Miller N.C.E. 1952. A new species of Afrius (Hem., Pentatomidae) predacious on Schematiza cordiae Barb., in Mauritius. Bulletin of Entomological Research 42: 183-184.

More S.V., Prashant M.S., Kadolkar S. \& Kesarkar V. 2017. Polymorphism in the southern green stink bug, Nezara viridula (L.) (Hemiptera: Pentatomidae) from Chandgad Tehsil. International Journal of Zoology Studies 2 (6): 239-241.

Mugo H.M. 1995. A Review of Insect Pests attacking Coffee Roots, Stems, Branches and Leaves in Kenya. Kenya Coffee 60 (708): 2095-2102.

Nagel P. \& Schmidlin L. 2014. Silbermann's "Revue entomologique": publication dates for nomenclatural purposes and bibliographic notes (Insecta, mainly Coleoptera). Zootaxa 3794 (1): 87-107. https://doi.org/10.11646/zootaxa.3794.1.3

Nonveiller G. 1984. Catalogue commenté et illustré des insectes du Cameroun d'intéret agricole (apparitions, répartitions, importance). Institut pour la Protection des Plantes, Beograd.

Nuamah K.A. 1982. Karyotypes of some Ghanaian shield-bugs and the higher systematics of the Pentatomoidea (Hemiptera Heteroptera). International Journal of Tropical Insect Science 3 (1): 9-28. https://oi.org/10.1017/S1742758400001843

Ohno K. \& Alam M.Z. 1992. Hereditary basis of adult colour polymorphism in the southern green stink bug, Nezara viridula Linné (Heteroptera: Pentatomidae). Applied Entomology and Zoology 27 (1): 133-139. https://doi.org/10.1303/aez.27.133 
Orian A.J.E. 1956. LXXX.- - Hemiptera (Heteroptera and Homoptera excluding Sternorrhyncha) of Mauritius. Annals and Magazine of Natural History 9 (105): 641-654.

https://doi.org/10.1080/00222935608655871

Orian A.J.E. 1965. Hemiptera of Mauritius. PhD Thesis, Imperial College London, England. Available from https://spiral.imperial.ac.uk/handle/10044/1/17749 [accessed 13 May 2018].

Paleari L.M. 2013. Developmental biology, polymorphism and ecological aspects of Stiretrus decemguttatus (Hemiptera, Pentatomidae), an important predator of cassidine beetles. Revista Brasileira de Entomologia 57 (1): 75-83. https://doi.org/10.1590/S0085-56262013000100012

Parra J.R.P., Botelho P.S.M., Corrêa-Ferreira B.S. \& Bento J.M. 2002. Controle biológico: Terminologia. In: Parra J.R.P., Botelho P.S.M., Corrêa-Ferreira B.S. \& Bento J.M. (eds) Controle biológico no Brasil: Parasitoides e Predadores: 1-17. Manole, Piracicaba.

Poutouli W., Silvie P. \& Aberlenc H.P. 2011. Hétéroptères phytophages et prédateurs d'Afrique de l'Ouest. Quae, Versailles/CTA, Wageningen.

Quicke D.L.J., Ingram S.N., Proctor J. \& Huddleston T. 1992. Batesian and Müllerian mimicry between species with connected life histories, with a new example involving braconid wasp parasites of Phoracantha beetles. Journal of Natural History 26: 1013-1034. https://doi.org/10.1080/00222939200770601

Rebagliati P.J., Mola L.M., Papeschi A.G. \& Grazia J. 2005. Cytogenetic studies in Pentatomidae (Heteroptera): A review. Zoological Systematics and Evolutionary Research 43 (3): 199-213. https://doi.org/10.1111/j.1439-0469.2005.00312.x

Renou A. 2007. Importance et Contrôle des Ravageurs en Culture cotonnière au Mali. CIRAD, Montpellier. Available from http://agritrop.cirad.fr/567196/1/document_567196.pdf [accessed 13 May 2018].

Reuter O.M. 1882. Ad cognitionem Heteropterorum Africae occidentalis. Öfversigt af Finska VetenskapsSocietetens Förhandlingar 25: 1-43.

Rider D.A. 2006. Family Pentatomidae. In: Aukema B. \& C. Rieger (eds) Catalogue of the Heteroptera of the Palaearctic Region. Vol. 5: Pentatomomorpha II: 233-402. The Netherlands Entomological Society, Amsterdam.

Risbec J. 1950. I - La Faune entomologique des Cultures au Sénégal et au Soudan français II Contribution à l'étude des Proctotrupidae. Gouvernement général de l'Afrique occidentale française, Dakar.

Robertson I.A.D. 2009. The Pentatomoidea (Hemiptera: Heteroptera) of Sub-Saharan Africa: a database. [s.n.], Malindi. Available from http://www.repository.naturalis.nl/document/228798 [accessed 9 May 2018].

Rolston L.H., Aalbu R.L., Murray M.J. \& Rider D.A. 1993. A Catalog of the Tessaratomidae of the World. Papua New Guinea Journal of Agriculture, Forestry \& Fisheries 36 (2): 36-108.

Rolston L.H., Rider D.A., Murray M.J. \& Aalbu R.L. 1996. Catalog of the Dinidoridae of the World. Papua New Guinea Journal of Agriculture, Forestry \& Fisheries 39 (1): 22-101.

Ruta R. \& Libonatti M.L. 2016. Redescriptions of Scirtidae (Coleoptera: Scirtoidea) described by Carl Henrik Boheman (1796-1868) with notes on Scirtes adustus diversenotatus Pic, 1930. Zootaxa 4072 (2): 203-216. https://doi.org/10.11646/zootaxa.4072.2.4

Schaefer C.W. 1996. Bright bugs and bright beetles: asopine pentatomids (Hemiptera: Heteroptera) and their prey. In: Alomar O. \& Wiedenmann R.N. (eds) Zoophytophagous Heteroptera: Implications for Life History and Integrated Pest Management: 18-56. Entomological Society of America, Lanham.

Scholtz C.H. \& Holm E. (eds) 1985. Insects of Southern Africa. Butterworths, Durban. 
Schouteden H. 1905a. Rhynchota Aethiopica II. Arminae et Tessaratominae. Faune entomologique de l'Afrique tropicale. Annales du Musée du Congo belge (Zoologie) 3 (1): 133-277.

Schouteden H. 1905b. Expédition du Baron C. von Erlanger en Abyssinie et au pays des Somalis. Hémiptères-Hétéroptères. Annales de la Société entomologique de Belgique 49: 11-16.

Schouteden H. 1907a. Heteroptera, Fam. Pentatomidae, Subfam. Asopinae (Amyoteinae). Genera Insectorum 52, P. Wytsman, Bruxelles.

Schouteden, H. 1907b. [Communication on the dating of Germar's "Hemiptera Heteroptera Promontorii Bonae Spei"]. Annales de la Société entomologique de Belgique 51: 7-8.

Schouteden H. 1909. Catalogues raisonnés de la faune entomologique du Congo Belge. I. HémiptèresHétéroptères, Fam. Pentatomidae. Annales du Musée du Congo belge (Zoologie) (3) 2 (1): 1-85.

Schouteden H. 1910. Wissenschaftliche Ergebnisse der schwedischen zoologischen Expedition nach dem Kilimandjaro, dem Meru und den Umgebenden Massaisteppen Deutsch-Ostafrikas 1905-1906 unter Leitung von Prof. Dr. Yngve Sjöstedt. 12. Hemiptera. 6. Pentatomidae. In: Sjöstedts KilimandjaroMeru Expedition 12: 73-96. Stockholm.

Schouteden H. 1911. Cimicidae et Coreidae recueillis dans les districts du Kasai et du Kwango par MM. Carlier, Koller et Luja. Revue zoologique africaine 1: 179-189.

Schouteden H. 1913a. Cimicidae et Coreidae recueillis au Congo par le Dr. J. Bequaert. Revue zoologique africaine 2 (2): 189-202.

Schouteden H. 1913b. Pentatomides de la Guinée Portugaise. Revue zoologique africaine 3 (2): 324 328.

Schouteden H. 1963. Pentatomides de la Côte d'Ivoire I. Revue de Zoologie et de Botanique africaines 68 (3-4): 397-402.

Schouteden H. 1964. Pentatomides de la Côte d'Ivoire II. Revue de Zoologie et de Botanique africaines 70 (1-2): 92-95.

Schouteden H. 1972. Pentatomidae. Exploration du Parc National de l'Upemba-Mission G.F. de Witte 72 (3): 83-113.

Schuh R.T. 1995. Plant Bugs of the World (Insecta: Heteroptera: Miridae): Systematic Catalog, Distributions, Host List, and Bibliography. New York Entomological Society, New York City.

Sherborn C.D. 1922-32. Index Animalium sive Index Nominum quae ab A. D. MDCCLVIII Generibus et Speciebus Animalium imposita sunt. Sectio secunda. A Kalendis Ianuariis, MDCCCI usque ad finem Decembris, MDCCCL. Longmans, Green \& Co. \& British Museum (Natural History), London.

Sherborn C.D. 1932. Index Animalium sive Index Nominum quae ab A.D. MDCCLVIII Generibus et Speciebus Animalium imposita sunt. Section 2, Part 28: INDEX variegatus-zizyphinus: 6807-7056. The Trustees of the British Museum, London.

Signoret V. 1861. Faune des Hémiptères de Madagascar. Pt. 2. Hétéroptères. Annales de la Société entomologique de France 8: 917-972.

Sileshi G., Maghembe J.A., Rao M.R., Ogol C.K.P.O. \& Sithanantham S. 2000. Insects feeding on Sesbania species in natural stands and agroforestry systems in southern Malawi. Agroforestry Systems 49: 41-52. https://doi.org/10.1023/A:1006378713271

Sileshi G., Kenis M., Ogol C.K.P.O. \& Sithanantham S. 2001. Predators of Mesoplatys ochroptera in sesbania planted-fallows in eastern Zambia. BioControl 46 (3): 289-310.

https://doi.org/10.1023/A:1011483320133 
Sileshi G., Mafongoya P.L., Kwesiga F.R., Kenis M., Rao M.R. \& Maghembe J.A. 2004. The pest spectrum of sesbania, Sesbania sesban (L.) Merrill, in eastern and southern Africa. In: Rao M.R. \& Kwesiga F.R. (eds) Proceedings of the Regional Agroforestry Conference on Agroforestry Impacts on Livelihoods in Southern Africa: putting Research into Practice: 245-255 [1-21]. World Agroforestry Centre (ICRAF), Nairobi.

Available from http://outputs.worldagroforestry.org/cgi-bin/koha/opac-detail.pl?biblionumber=37376 [accessed 17 May 2018].

Silvie P., Delvare G. \& Maldès J.-M. 1989. Arthropodes associés à la culture cotonnière au Tchad: ravageurs, prédateurs et parasites. Coton et fibres tropicales 44 (4): 275-290.

Singh-Pruthi H. 1925. The morphology of the male genitalia in Rhynchota. Transactions of the Entomological Society of London 127-267. https://doi.org/10.1111/j.1365-2311.1925.tb02861.x

Smith J.W. \& Barfield C.S. 1982. Management of preharvest insects. In: Pattee H.E. \& Young C.T. (eds) Peanut Science and Technology: 250-325. American Peanut Research and Education Society, Inc., Yoakum, Texas.

Smithsonian Institution. 2018. Search the Department of Entomology Collections.

Available from https://collections.nmnh.si.edu/search/ento/ [accessed 30 May 2018].

Stål C. 1854. Nya Hemiptera från Cafferlandet. Öfversigt af Kongliga Vetenskaps-Akademiens Förhandlingar 10 (9): 209-227.

Stål C. 1862. Synonymiska och systematiska anteckningar öfver Hemiptera. Öfversigt af Kongliga Vetenskaps-Akademiens Förhandlingar 19 (9): 479-504.

Stål C. 1864. Hemiptera Africana, vol. 1. Norstedt, Stockholm. https://doi.org/10.5962/bhl.title.8566

Stål C. 1870. Enumeratio Hemipterorum. Bidrag till en förteckning öfver alla hittills kända Hemiptera, jemte systematiska meddelanden. Kongliga Svenska Vetenskaps-Akademiens Handlingar 9 (1): 1-232. https://doi.org/10.5962/bhl.title.12549

Tchibozo S \& Braet Y. 2004. Note péliminaire sur les insectes terrestres de la forêt classée de la Lama et de ses alentours (République du Bénin). Bulletin de la Société royale d'Entomologie 140: 157-162.

Thomas D.B. 1992. Taxonomic synopsis of the Asopine Pentatomidae (Heteroptera) of the Western Hemisphere. The Thomas Say Foundation, Monographs 16, Entomological Society of America.

Thomas D.B. 1994. Taxonomic synopsis of the Old World asopine genera Heteroptera: Pentatomidae). Insecta Mundi 8: 145-211.

Van Harten A. 1993. Terrestrial arthropods of the Cape Verde Islands. A check-list. In: Lobin W. (ed.) Beitrag zur Fauna und Flora der Kapverdischen Inseln 9. Ergebnisse des 5. Symposiums in Leiden: 235310. Courier Forschungsinstitut Senckenberg 159, Senckenbergische Naturforschende Gesellschaft, Frankfurt am Main.

Van Heerden P.W. 1931. The Pentatomidae of South Africa. Msc Thesis. Stellenbosch University, South Africa. Available from http://scholar.sun.ac.za/handle/10019.1/50772 [accessed 14 May 2018].

Villiers A. 1949. Mission P.L. Dekeyser et A. Villiers en Guinée et Côte-d'Ivoire (1946). Insectes (Première partie). Institut français d'Afrique noire, Catalogues 5: 1-90.

Villiers A. 1952a. Mission A. Villiers au Togo et au Dahomey (1950). XXI. Hémiptères. Bulletin de l'Institut français d'Afrique noire 14 (4): 1196-1213.

Villiers A. 1952b. Hémiptères de l'Afrique noire (Punaises et Cigales). Initiations africaines 9, Institut français d'Afrique noire, Dakar. 
Villiers A. 1954. Contribution à l'étude du peuplement de la zone d'inondation du Niger (Mission G. Remaudière). X. - Hémiptères Hétéroptères. Bulletin de l'Institut français d'Afrique noire 16 (1): 219231.

Villiers A. 1956. Voyage de J. Bechyné en Afrique occidentale. Hemiptera, Pentatomidae et Coptosomatidae. Entomologische Arbeiten aus dem Museum G. Frey 7: 203-216.

Vivan L.M. \& Panizzi A.R. 2002. Two new morphs of the southern green stink bug, Nezara viridula (L.) (Heteroptera: Pentatomidae), in Brazil. Neotropical Entomology 31 (3): 475-476. https://doi.org/10.1590/S1519-566X2002000300020

Vuillet A. \& Vuillet J. 1912. Notes sur les insectes nuisibles au karité. L'Agriculture pratique des Pays chauds 12 (2): 436-448.

Vuillet J. \& Vuillet A. 1911. Notes sur Cerina Butyrospermi A. Vuillet. Insecta 1: 271-277.

Wallengren H.D.J. 1875. Insecta Transvaaliensia - Bidrag till Transvaalska Republikens i Södra Afrika Insektfauna. Öfversigt af Kongliga Vetenskaps-Akademiens Förhandlingar 32 (1): 83-137.

Westwood J.O. 1837. A Catalogue of Hemiptera in the Collection of Rev. F.W. Hope, M.A., with Short Latin Diagnoses of the New Species. J.C. Bridgewater, London.

Williams J.R. 1951. The control of the black sage in Mauritius by Schematiza cordiae Barb. (Col., Galerucid.). Bulletin of Entomological Research 42 (2): 455-463.

https://doi.org/10.1017/S0007485300025451

Zacher F. 1921. Schädlinge der Nutzpflanzen im West Sudan. Der Tropenpflanzer; Zeitschrift für tropische Landwirtschaft 24: 132-142 [With an English summary in The Review of Applied Entomology (A) 10 (1922): 27-28.].

Zhao Q., Rédei D. \& Bu W. 2013. A revision of the genus Pinthaeus (Hemiptera: Heteroptera: Pentatomidae). Zootaxa 3636 (1): 59-84. https://doi.org/10.11646/zootaxa.3636.1.3

Zucchi R.A. 2002. A taxonomia e o controle biológico de pragas. In: Parra J.R.P., Botelho P.S.M., Corrêa-Ferreira B.S. \& Bento J.M. (eds) Controle biológico no Brasil: Parasitoides e Predadores: 1727. Manole, São Paulo.

Manuscript received: 15 June 2018

Manuscript accepted: 23 November 2018

Published on: 24 April 2019

Topic editor: Gavin Broad

Section editor: Anna Namyatova

Desk editor: Kristiaan Hoedemakers

Printed versions of all papers are also deposited in the libraries of the institutes that are members of the EJT consortium: Muséum national d'Histoire naturelle, Paris, France; Meise Botanic Garden, Belgium; Royal Museum for Central Africa, Tervuren, Belgium; Royal Belgian Institute of Natural Sciences, Brussels, Belgium; Natural History Museum of Denmark, Copenhagen, Denmark; Naturalis Biodiversity Center, Leiden, the Netherlands; Museo Nacional de Ciencias Naturales-CSIC, Madrid, Spain; Real Jardín Botánico de Madrid CSIC, Spain; Zoological Research Museum Alexander Koenig, Bonn, Germany. 